\title{
THE FREE PATH IN A HIGH VELOCITY RANDOM FLIGHT PROCESS ASSOCIATED TO A LORENTZ GAS IN AN EXTERNAL FIELD
}

\begin{abstract}
ALEXANDRU HENING, DOUGLAS RIZZOLO, AND ERIC S. WAYMAN
Abstract. We investigate the asymptotic behavior of the free path of a variable density random flight model in an external field as the initial velocity of the particle goes to infinity. The random flight models we study arise naturally as the Boltzmann-Grad limit of a random Lorentz gas in the presence of an external field. By analyzing the time duration of the free path, we obtain exact forms for the asymptotic mean and variance of the free path in terms of the external field and the density of scatterers. As a consequence, we obtain a diffusion approximation for the joint process of the particle observed at reflection times and the amount of time spent in free flight.
\end{abstract}

\section{Contents}

1. Introduction

2. The free path of the particle

3. Convergence of the free path process

4. The process on its natural time scale

5. Classifying the boundaries of $\mathcal{D}$

6. Higher dimensions and general potentials

Acknowledgments

References

\section{INTRODUCTION}

We study the behavior of a random flight process that arises as the BoltzmannGrad limit of a random scatterer Lorentz gas with an external field. The Lorentz gas model was introduced in 1905 by H. A. Lorentz Lor05 as a model for the motion of electrons in metallic bodies. Since its introduction, the model has been widely studied by both mathematicians and physicists (see Det14 for a recent survey). In this model, a point particle travels in an array of fixed convex scatterers. When the particle comes in contact with a scatterer it reflects specularly. This variant is referred to as the "hard core" version. There is also a "soft core" version in which the scatterers deflect the moving particle via a potential instead of a hard boundary. Of the many variations on the hard core model, our study is motivated

Received by the editors March 8, 2015.

2010 Mathematics Subject Classification. Primary 60F17; Secondary 60J60, 82C70.

Key words and phrases. Random flight process, Lorentz gas, random evolution, transport process, continuous time random walk, diffusion approximation.

The first author was supported by EPSRC grant EP/K034316/1.

The second author was supported by NSF grant DMS-1204840. 
by the version in which an array of spherical scatterers is chosen randomly and the flight of the moving particle is determined by the action of an external field. The random scatterer Lorentz gas is difficult to study directly even in the absence of an external field. One approach that has proven tractable, however, is the BoltzmannGrad limit. The Boltzmann-Grad limit is a low density limit in which the number of scatterers in a fixed box goes to infinity while, at the same time, the size of each scatterer goes to zero in such a way that the total volume of the scatterers in the box goes to zero. If the centers of scatterers are placed according to a Poisson process and the rates are chosen appropriately, the asymptotic behavior of the moving particle is described by a Markovian random flight process Gal78, Spo78, Spo88. The Markovian nature of the Boltzmann-Grad limit is due to the following two observations: re-collisions with scatterers become unlikely as the size of each scatterer goes to zero, and the Poisson nature of the scatterer locations which means that knowing the location of one scatterer does not give information about the locations of the other scatterers. Since analyzing the random Lorentz gas directly is beyond the capability of current techniques, this random flight model is commonly studied in both the mathematics literature [RT99, Vys06, BFS00, BCLDM02, BR14 and the physics literature ADBLP10, dWB04, vBM05, MvB04 to gain insight into the behavior of random Lorentz gas models.

We are interested in the regime in which the particle's velocity is (typically) large. There are several natural examples of this setting. The first is an external field which accelerates the particle towards infinity. For example, the influence of a constant gravitational field has been studied for the random flight process in both a constant density of scatterers PW79, RT99, a variable density of scatterers [BR14, and for the periodic Lorentz gas in two dimensions CD09. The random flight model in a gravitational field has also been used as a model for a particle percolating through a porous medium WE82. A second example of a high velocity limit is particle motion in a centered, mean zero, isotropic force field [DK09, KR06]. The soft core Poisson Lorentz gas is an example of this situation. A discussion of how much of the past work on particles moving in random force fields fits into the high velocity framework can be found in DK09.

A unique contribution of this work is that we study fields that are in general not mean zero, unlike prior work on random force fields. For example, the constant field directed towards the origin fits into our setting. In such fields, conservation of energy implies that particles have bounded trajectories. We are interested in the behavior of the system as the velocity of the particle, or equivalently the energy of the system, is sent to infinity. Our primary focus is to study the free path of the particle, which is the path of the particle between two reflection times. The study of the free path has played a key role in much of the work on the hard core Lorentz gas. To highlight a few examples, the free path played an important role in the recent derivation of the Boltzmann-Grad limit of the periodic Lorentz gas MS11a, MS11b, which is substantially more complicated than the corresponding result for the random Lorentz gas. Furthermore, it has also played a central role in the derivation of a diffusive limit for the two-dimensional periodic Lorentz gas in a gravitational field [CD09]. For these reasons, the free path has become an object of interest in its own right; see e.g. MS10, MS14a, MS14b, Wen12, BZ07, BGW98, GW00 CG03. We determine precisely the asymptotic behavior of the free path of a particle whose domain of motion is predominantly in the high velocity regime. 
The primary complication that arises in our study is that the domain of the particle is determined by the external field and, as a result, the particle's velocity is not bounded away from zero. If the particle enters a part of the domain where it is moving slowly, then there can be a long time before a reflection occurs, and we must control this in order to analyze the asymptotic behavior of the mean free path. As a consequence of our analysis of the free path, we obtain several diffusion approximations that illustrate different aspects of the particle's behavior.

We remark that our techniques may be of interest beyond the study of the Boltzmann-Grad limit of a Lorentz gas. The random flight process we study falls into a general class of models known as transport process. Models similar to the ones we study here are used in many areas of physics and chemistry as microscopic models for particle behavior. For example, they arise in neuron transport AB99, the study of semiconductors BAD96], electron transfer dynamics HW13, WW05, and numerous other fields [BR00, Deg98, DGL81, DLLS13, $\mathrm{HLB}^{+} 09$ ].

1.1. The model. We are primarily interested in the process in dimension three, which is the most interesting spatial dimension and also a dimension where certain technical simplifications occur. Let us start with a more detailed description of the Boltzmann-Grad limit. Let $\mathcal{U}: \mathbb{R}^{3} \rightarrow \mathbb{R}$ and $g: \mathbb{R}^{3} \rightarrow(0, \infty)$ be differentiable. The function $\mathcal{U}$ will serve as the potential for a conservative force and $g$ will be the density of scatterers. Fix an energy level $E$ and consider a particle moving in the potential $\mathcal{U}$ with total energy $E$. By conservation of energy

$$
\frac{m\|\mathbf{v}(t)\|^{2}}{2}+\mathcal{U}(\mathbf{y}(t))=E
$$

where $\mathbf{y}$ is the particle's position and $\mathbf{v}$ is its velocity. In this setting, the BoltzmannGrad limit can be obtained as follows. Assume spherical scatterers with radius $1 / R$ are placed so their centers are the points of a Poisson process with intensity $R^{2} g$. Further assume that for almost every initial condition the trajectory of a particle moving in the potential $\mathcal{U}$ with total energy $E$ is open and has infinite length. The arguments of [Spo78, Spo88 can easily be adapted to include the external field and produce the following result: if the initial position and velocity of the particle is absolutely continuous with respect to Lebesgue measure on the constant energy surface, then the distribution of the position and velocity process of the particle converges as $R \rightarrow \infty$, in the sense of convergence of finite-dimensional distributions, to a Markovian random flight process $(\mathbf{X}(t), \mathbf{V}(t))_{t \geq 0}$ with generator

$$
\begin{aligned}
D f(\mathbf{x}, \mathbf{v})=\mathbf{v} \cdot \nabla_{\mathbf{x}} f(\mathbf{x}, \mathbf{v})-\frac{1}{m} & \nabla_{\mathbf{x}} \mathcal{U} \cdot \nabla_{\mathbf{v}} f(\mathbf{x}, \mathbf{v}) \\
& +g(\mathbf{x})\|\mathbf{v}\| \int_{\mathbf{S}^{2}}(f(\mathbf{x},\|\mathbf{v}\| \mathbf{u})-f(\mathbf{x}, \mathbf{v})) \sigma(d \mathbf{u}),
\end{aligned}
$$

where $\sigma$ is the normalized surface measure on the unit sphere $\mathbf{S}^{2}:=\{\mathbf{x}:\|\mathbf{x}\|=1\}$; see Spo88. A similar result holds in dimensions other than three, but instead of the integral being against the normalized surface measure it is against a kernel that depends on $\mathbf{v}$.

Remark 1.1. The assumption that for almost every initial condition the trajectory of a particle with mass $m$ moving in the potential $\mathcal{U}$ with total energy $E$ is open and has infinite length is essential to the derivation of the Boltzmann-Grad limit. The intuition for its necessity is that it is possible for a periodic orbit to avoid 
all of the scatterers in a random arrangement. If this happens, the effect on the limiting process is that one must keep track of whether or not an orbit has closed, which creates long range dependencies in the limiting process. The effect of this phenomenon on the Boltzmann-Grad limit has been the subject of study in both the mathematics and physics literature BHHP96, BMHH97, BHPH01, DR04, KS98. In these cases one may still obtain a limiting process with generator of the form (1.1), but additional randomness must be added to the array of scatterers. For example, they can be allowed to move (but not transfer energy), or they can be arranged according to a space-time Poisson process where a scatterer comes into existence, exists for a certain amount of time, and then disappears.

The process $(\mathbf{X}(t), \mathbf{V}(t))_{t \geq 0}$ can be constructed in the following way, which explains the name "random flight process". Let $\left(\mathbf{y}\left(\mathbf{x}_{0}, \mathbf{v}_{0}, t\right)\right)_{t \geq 0}$ with $\mathbf{x}_{0}, \mathbf{v}_{0} \in \mathbb{R}^{3}$ and $m\left\|\mathbf{v}_{0}\right\|^{2} / 2+\mathcal{U}\left(\mathbf{x}_{0}\right)=E$ denote the solution to the initial value problem

$$
\left\{\begin{array}{llr}
m \mathbf{y}^{\prime \prime} & = & -\nabla \mathcal{U}(\mathbf{y}), \\
\mathbf{y}(0) & = & \mathbf{x}_{0}, \\
\mathbf{y}^{\prime}(0) & = & \mathbf{v}_{0} .
\end{array}\right.
$$

We construct our process $((\mathbf{X}(t), \mathbf{V}(t)), t \geq 0)$ recursively as follows. Set $(\mathbf{X}(0), \mathbf{V}(0))=\left(\mathbf{x}_{0}, \mathbf{v}_{0}\right)$ and let $T_{0}=0$. For $k \geq 1$, assuming we have defined $((\mathbf{X}(t), \mathbf{V}(t)))_{0 \leq t \leq T_{k-1}}$, let $\mathbf{U}_{k-1}$ be independent of this part of the path and uniformly distributed on $\mathbf{S}^{2}$ and let $T_{k}$ satisfy

$$
\begin{aligned}
& \mathbb{P}\left(T_{k}-T_{k-1}>t \mid \mathbf{U}_{k-1},\left(\left(\mathbf{X}_{t}, \mathbf{V}_{t}\right)\right)_{0 \leq t \leq T_{k-1}}\right) \\
&=\exp \left(-\int_{0}^{t} g\left(\mathbf{y}\left(\mathbf{X}\left(T_{k-1}\right),\left\|V\left(T_{k-1}\right)\right\| \mathbf{U}_{k-1}, s\right)\right)\right. \\
&\left.\quad \times\left\|\mathbf{y}^{\prime}\left(\mathbf{X}\left(T_{k-1}\right),\left\|\mathbf{V}\left(T_{k-1}\right)\right\| \mathbf{U}_{k-1}, s\right)\right\| d s\right) .
\end{aligned}
$$

For $t \in\left[T_{k-1}, T_{k}\right]$ we then define

$$
\begin{aligned}
& \mathbf{X}(t):=\mathbf{y}\left(\mathbf{X}\left(T_{k-1}\right),\left\|\mathbf{V}\left(T_{k-1}\right)\right\| \mathbf{U}_{k-1}, t-T_{k-1}\right) \\
& \mathbf{V}(t):=\mathbf{y}^{\prime}\left(\mathbf{X}\left(T_{k-1}\right),\left\|\mathbf{V}\left(T_{k-1}\right)\right\| \mathbf{U}_{k-1}, t-T_{k-1}\right)
\end{aligned}
$$

We remark that under very mild assumptions $T_{k} \rightarrow \infty$ a.s. and thus this defines the path of the particle for all times. Intuitively, $T_{k}$ defines the $k$ th reflection of our particle by a scatterer. By studying the free path of the particle, we mean to study, in particular, the conditional law of $(\mathbf{X}(t))_{T_{1} \leq t \leq T_{2}}$ given $\mathbf{X}\left(T_{1}\right)=\mathbf{x}$ under an appropriate scaling of the parameters in the model. Note that the path of the particle on the time interval $\left[T_{1}, T_{2}\right]$ is the path between two consecutive reflections. This is why we call it the free path.

At this point we make a simple but important observation. By conservation of energy,

so that, if we define

$$
\|\mathbf{V}(t)\|=\sqrt{\frac{2}{m}(E-\mathcal{U}(\mathbf{X}(t)))}
$$

$$
v(\mathbf{x})=\sqrt{\frac{2}{m}(E-\mathcal{U}(\mathbf{x}))},
$$


then

$$
\mathbf{X}(t)=\mathbf{y}\left(\mathbf{X}\left(T_{k-1}\right), v\left(\mathbf{X}\left(T_{k-1}\right)\right) \mathbf{U}_{k-1}, t-T_{k-1}\right) .
$$

Since $\mathbf{U}_{k-1}$ is independent of $((\mathbf{X}(t), \mathbf{V}(t)))_{0 \leq t \leq T_{k-1}}$, this implies that if we define $\mathbf{X}_{k}=\mathbf{X}\left(T_{k}\right)$, then $\left(\mathbf{X}_{k}\right)_{k \geq 1}$ is a Markov chain. That the index in this chain starts at 1 is an artifact of our deterministic choice of $\mathbf{V}(0)$. If instead of choosing $\mathbf{V}(0)=\mathbf{v}_{0}$ in the construction above we took $\mathbf{V}(0)=v(\mathbf{X}(0)) \mathbf{U}$, with $\mathbf{U}$ uniformly distributed on $\mathbf{S}^{2}$, then $\left(\mathbf{X}_{k}\right)_{k \geq 0}$ is a Markov chain and its transition operator is

$$
P f(\mathbf{x})=\mathbb{E}[f(\mathbf{y}(\mathbf{x}, v(\mathbf{x}) \mathbf{U}, N(\mathbf{x}, \mathbf{U})))],
$$

where $N(\mathbf{x}, \mathbf{u})$ is a random variable with distribution

$$
\mathbb{P}(N(\mathbf{x}, \mathbf{u})>t)=\exp \left(-\int_{0}^{t} g[\mathbf{y}(\mathbf{x}, v(\mathbf{x}) \mathbf{u}, s)] v[\mathbf{y}(\mathbf{x}, v(\mathbf{x}) \mathbf{u}, s)] d s\right),
$$

and, conditional on $\mathbf{U}=\mathbf{u}, N(\mathbf{x}, \mathbf{U})$ is distributed like $N(\mathbf{x}, \mathbf{u})$.

In order to avoid the notational difficulties and technical assumptions on the geometry of the set $\left\{\mathbf{x} \in \mathbb{R}^{3}: \mathcal{U}(\mathbf{x})=E\right\}$ that come from working with a general potential $\mathcal{U}$, we restrict ourselves to the spherically symmetric case where $\mathcal{U}(\mathbf{x})=$ $\mathcal{U}(r)$ and $g(\mathbf{x})=g(r)$, where $r=\|\mathbf{x}\|$. In this case angular momentum is conserved, which implies that the motion of the particle between collisions with scatterers takes place in a plane. Consequently, we may restrict our attention to $\mathbb{R}^{2}$ for some of the analysis of the free path. This lets us work in polar coordinates for these aspects of the analysis. Throughout let $\mathbb{R}_{+}:=[0, \infty), \mathbb{N}_{0}:=\{0,1,2, \ldots\}=\mathbb{N} \cup\{0\}$, and let $\mathcal{C}^{k}(S)$ be the set of real-valued functions on $S$ having $k$ continuous derivatives. We let $\mathcal{U}(r)$ be the potential and we assume our particle has mass $m$ and total energy $E$. Let $\mathbf{r}(t):=\left(\left(r\left(r_{0}, \theta, t\right), \alpha\left(r_{0}, \alpha_{0}, \theta, t\right)\right), t \geq 0\right)$ denote the trajectory in polar coordinates of a particle with mass $m>0$, total energy $E$, initial position $\left(r_{0}, \alpha_{0}\right)$, and whose velocity vector at time 0 makes an angle $\theta$ with the radial vector. Consequently $\mathbf{r}(t)$ is the solution to the equations of motion in polar coordinates for a particle of mass $m$ in the potential $\mathcal{U}$ :

$$
\frac{1}{r} \frac{d}{d t}\left(r^{2} \dot{\alpha}\right) e_{\alpha}+\left(\ddot{r}-r \dot{\alpha}^{2}\right) e_{r}=-\frac{\partial_{r} \mathcal{U}(r)}{m} e_{r}
$$

with initial conditions

$$
\begin{aligned}
\mathbf{r}(0) & =\left(r_{0}, \alpha_{0}\right), \\
\dot{r}(0) & =v\left(r_{0}\right) \cos \theta, \\
\dot{\alpha}(0) & =\frac{v\left(r_{0}\right)}{r_{0}} \sin \theta,
\end{aligned}
$$

where $e_{r}$ is the radial unit vector, $e_{\alpha}$ is the angular unit vector, and $\theta$ is the angle the initial velocity makes with the line segment from the origin to the point $\mathbf{r}(0)$. We remark that in this setup it is generally possible for the trajectory to hit the origin in finite time, at which point the equations of motion are no longer well defined. In this case we continue the path in such a way that momentum is continuous, if possible, and such that angular momentum is conserved. The extension is most easily described in Euclidean coordinates, since it may involve the angular component re-entering from $\pm \infty$ in polar coordinates. Suppose that $\mathbf{y}(t)$ is a trajectory that hits the origin for the first time at time $T>0$. We extend the path to the interval $[T, 2 T]$ by defining $\mathbf{y}(t)=-\mathbf{y}(2 T-t)$ for $t \in[T, 2 T]$. Since $\|\mathbf{y}(2 T)\|=\|\mathbf{y}(0)\| \neq 0$, we can continue the path by the equations of motion 
until it hits the origin again, at which point we repeat the extension procedure. A straightforward calculation using the radial symmetry of the potential shows that

$$
\frac{d^{2}}{d t^{2}}(-\mathbf{y}(2 T-t))=-\Delta \mathcal{U}(-\mathbf{y}(2 T-t))
$$

so our extension solves (1.2) away from the origin and it is easy to see that $\mathbf{y}^{\prime}$ is continuous at $T$ if $\lim _{t \uparrow T} y^{\prime}(t)$ exists. In this way, we guarantee that all initial conditions lead to trajectories that exist for all time.

Let $\bar{v}(t):=v(r(t))$ denote the speed of the particle as a function of time. By conservation of energy, we have

$$
\frac{m v^{2}(r)}{2}+\mathcal{U}(r)=E
$$

and, as a result,

$$
v(r)=\sqrt{\frac{2}{m}(E-\mathcal{U}(r))} .
$$

We can also write $\bar{v}(t)$ as a function of angular and radial velocity:

$$
\bar{v}(t)=\sqrt{\dot{r}^{2}(t)+r^{2}(t) \dot{\alpha}^{2}(t)} .
$$

1.2. Heuristics. In this section, we give heuristic arguments to motivate our scaling. Consider the following simple situation that encapsulates the phenomena in which we are interested. In particular, suppose that $m=2, \mathcal{U}(\mathbf{x})=\|\mathbf{x}\|$, so that $\mathcal{U}$ produces a uniform acceleration towards the origin, and that $g(\mathbf{x}) \equiv 1$. Suppose we start the particle at the origin with speed $v_{0}=1$ moving in direction $\mu$. By conservation of energy the total energy of the particle will be

$$
E\left(v_{0}\right)=m v_{0}^{2} / 2=1 .
$$

Since $\mathcal{U}$ produces a uniform acceleration towards the origin, the maximum distance the particle can move away from the origin is 1 . Consequently, there is no way to rescale the trajectory of the particle to obtain a diffusive limit: Any scaling of space will cause the trajectory to degenerate, and scaling time alone will produce jumps. However, we can obtain a diffusive limit if we let $v_{0}$ (and, consequently, $E$ ) go to infinity. This is the type of high velocity limit considered in DK09, KR06]. In those papers, however, the high velocity was used to prevent the particle from being trapped in a bounded domain, while in our setting the particle is still trapped in a bounded domain, but the size of the domain goes to infinity as $v_{0}$ does.

Treating $v_{0}$ as a parameter, the energy becomes a function of $v_{0}$, specifically $E\left(v_{0}\right)=v_{0}^{2}$ is the maximum distance the particle can travel from the origin. Let $\left(\mathbf{X}^{v_{0}}(t)\right)_{t \geq 0}$ be the trajectory of the random flight process with initial conditions $\mathbf{X}^{v_{0}}(0)=\mathbf{0}$ and initial velocity $\dot{\mathbf{X}}^{v_{0}}(0)=v_{0} \mathbf{U}$, where $\mathbf{U}$ is uniformly distributed on $\mathbf{S}^{2}$. In order to obtain a diffusive limit for $\left(\mathbf{X}^{v_{0}}(t)\right)_{t \geq 0}$, we rescale space so that its maximum distance from the origin remains constant. In particular, we look at $\left(v_{0}^{-2} \mathbf{X}^{v_{0}}(t)\right)_{t \geq 0}$, which travels at most distance 1 from the origin. This is the process for which we analyze the behavior of the free paths.

Since the velocity of the particle is going to infinity, the time between reflections is going to 0 , so we can expect a diffusive limit if we scale time appropriately. Since $g \equiv 1$, the distance that $\left(\mathbf{X}^{v_{0}}(t)\right)_{t \geq 0}$ travels between reflections has an exponential distribution with rate 1 , so the distance that $\left(v_{0}^{-2} \mathbf{X}^{v_{0}}(t)\right)_{t \geq 0}$ travels between reflections is of order $v_{0}^{-2}$. Writing down the equations of motion for $\left(v_{0}^{-2} \mathbf{X}^{v_{0}}(t)\right)_{t \geq 0}$ 
between reflections, we find that its trajectories are the same as those governed by the potential $\mathcal{U}^{v_{0}}(\mathbf{x})=v_{0}^{-2} \mathcal{U}(\mathbf{x})$ moving with total energy $E^{v_{0}}=v_{0}^{-2}$ and initial velocity $v_{0}^{-1}$, and the scattering rate as measured along these paths is $g^{v_{0}}(\mathbf{x})=v_{0}^{2}$.

By Brownian scaling, in order to obtain a diffusive limit, we expect that we should scale time so that there are approximately $v_{0}^{4}$ reflections per unit time. Typically, we expect the particle to be distance of order $v_{0}^{2}$ from the origin, and if $\mathbf{X}^{v_{0}}(t)=v_{0}^{2} \mathbf{x}$ and $t$ is a reflection time, the time until the next reflection is approximately

$$
\frac{1}{\left\|\dot{\mathbf{X}}^{v_{0}}(t)\right\|}=\frac{1}{\sqrt{v_{0}^{2}-v_{0}^{2}\|\mathbf{x}\|}}=\frac{1}{v_{0} \sqrt{1-\|\mathbf{x}\|}}
$$

Thus the amount of time for $v_{0}^{4}$ reflections to occur is of the order $v_{0}^{3}$. This suggests looking for a diffusive limit of $\left(v_{0}^{-2} \mathbf{X}^{v_{0}}\left(v_{0}^{3} t\right)\right)_{t \geq 0}$ as $v_{0}$ tends to infinity, which is what we undertake.

1.3. General scaling and assumptions. In general, we allow $g$ to be a small perturbation of the constant density and $\mathcal{U}$ to produce a small perturbation of the constant field, small being relative to the speed of the particle. In particular, we will assume the following conditions.

(A1) The path of the particle $\left(\mathbf{X}^{n}(t)\right)_{t \geq 0}$ evolves as the position of a random flight process where the density of scatterers is

$$
g_{n}:=\sqrt{n} g,
$$

the potential energy of the field is

$$
\mathcal{U}_{n}:=\frac{1}{\sqrt{n}} \mathcal{U}
$$

and the total energy of the particle is

$$
E_{n}:=\frac{1}{\sqrt{n}} E,
$$

where $g, \mathcal{U}$, and $E$ are fixed and independent of $n$ and $g(\mathbf{x})=g(\|\mathbf{x}\|)$ and $\mathcal{U}(\mathbf{x})=$ $\mathcal{U}(\|\mathbf{x}\|)$. That is, the density of scatterers and potential are spherically symmetric. By (1.12) we can write the speed as a function of $E$ and $\mathcal{U}$; consequently the speed $v$ is also rescaled as

$$
v_{n}(r):=\frac{1}{n^{1 / 4}} v(r) .
$$

The trajectory of the particle with these rescaled parameters is denoted by

$$
\left(\mathbf{y}_{n}\left(\mathbf{x}, v_{n}(\mathbf{x}) \mathbf{u}, t\right)\right)_{t \geq 0}
$$

when we are working with the path in three dimensions and by

$$
\left(\left(r_{n}\left(r_{0}, \theta, t\right), \alpha_{n}\left(r_{0}, \alpha_{0}, \theta, t\right)\right), t \geq 0\right)
$$

when we are considering the path in polar coordinates with respect to its plane of motion. When no confusion will arise, we will leave the dependence on initial conditions implicit.

In general, subscripts or superscripts of $n$ refer to distributions relative to these scaled parameters. For example, corresponding to (1.8) is the random variable $N^{(n)}(\mathbf{x}, \mathbf{u})$ with distribution

$$
\mathbb{P}\left(N^{(n)}(\mathbf{x}, \mathbf{u})>t\right)=\exp \left(-\int_{0}^{t} g_{n}\left[\mathbf{y}_{n}\left(\mathbf{x}, v_{n}(\mathbf{x}) \mathbf{u}, s\right)\right] v_{n}\left[\mathbf{y}_{n}\left(\mathbf{x}, v_{n}(\mathbf{x}) \mathbf{u}, s\right)\right] d s\right),
$$


and corresponding to (1.7) is the Markov chain $\left(\mathbf{X}_{k}^{n}\right)_{k \geq 0}$ with transition operator

$$
P_{n} f(\mathbf{x})=\mathbb{E}\left[f\left(\mathbf{y}_{n}\left(\mathbf{x}, v_{n}(\mathbf{x}) \mathbf{U}, N^{(n)}(\mathbf{x}, \mathbf{U})\right)\right)\right],
$$

where $\mathbf{U}$ is uniformly distributed on $\mathbf{S}^{2}$ and, conditionally given $\mathbf{U}=\mathbf{u}, N^{(n)}(\mathbf{x}, \mathbf{U})$ is distributed like $N^{(n)}(\mathbf{x}, \mathbf{u})$. Similarly, $\left(\mathbf{X}^{n}(t)\right)_{t \geq 0}$ will be constructed as in (1.6) using the rescaled parameters.

Remark 1.2. To make the connection with the heuristic arguments in Section 1.2 , if we take $v_{0}=n^{1 / 4}, g=1$, and $\mathcal{U}(\mathbf{x})=\|\mathbf{x}\|$, then $\left(\mathbf{X}^{n}(t)\right)_{t \geq 0}={ }_{d}\left(v_{0}^{-2} \mathbf{X}^{v_{0}}(t)\right)_{t \geq 0}$. Thus the scaling we do here accounts for increasing the initial speed and scaling space to keep the particle's trajectory contained. This is the scaling under which we analyze the free path of the particle. We will scale time separately when we consider diffusive limits.

(A2) We assume that for all $n \in \mathbb{N}$ the process $\left(\left\|\mathbf{X}^{n}(t)\right\|, t \geq 0\right)$ evolves in a domain $\mathcal{D} \subset \mathbb{R}_{+}$where $\mathcal{D}=\mathbb{R}_{+}$or $\mathcal{D}=\left[h_{-}, h_{+}\right], 0 \leq h_{-}<h_{+}<\infty$. The domain $\mathcal{D}$ is chosen so that $E-U(r)>0$ for all $r \in \mathcal{D}^{\circ}$. This is equivalent to $v(r)>0$ for all $r \in \mathcal{D}^{\circ}$.

We require the particle to have positive speed in the interior $\mathcal{D}^{\circ}$ so the time between reflections approaches 0 as $n$ goes to infinity. This way we obtain a nontrivial diffusion limit.

(A3) On the boundary $\partial \mathcal{D}$ we have the following assumptions.

- If $h_{-}>0$, then

$$
\begin{aligned}
\mathcal{U}\left(h_{-}\right) & =E \\
-\partial_{r} \mathcal{U}\left(h_{-}\right) & :=-\frac{\partial \mathcal{U}}{\partial r}\left(h_{-}\right)>0 .
\end{aligned}
$$

- If $h_{+}<\infty$, then

$$
\begin{aligned}
\mathcal{U}\left(h_{+}\right) & =E, \\
-\partial_{r} \mathcal{U}\left(h_{+}\right) & <0 .
\end{aligned}
$$

The conditions on $\mathcal{U}$ force the speed of the particle to be zero at the respective endpoints $h_{-}$and $h_{+}$, while the conditions on $\frac{\partial \mathcal{U}}{\partial r}$ ensure that the force field at the endpoints is pointing towards the interior $\mathcal{D}^{\circ}$. Having zero speed at the boundaries and having the force field point 'inwards' prevents the particle from leaving the domain $\mathcal{D}$.

- If $h_{-}=0$, then

$$
E-\mathcal{U}(0)>0 .
$$

This condition ensures the particle is not trapped at the origin.

- If $h_{+}=\infty$ we require that for any $\varepsilon>0$, $\inf _{\left[h_{-}+\epsilon, \infty\right)}(E-\mathcal{U}(r))>0$.

This condition implies that for any $\varepsilon>0, \inf _{\left[h_{-}+\epsilon, \infty\right)} v(r)>0$ which shows that the reflection rate does not go to 0 as the process goes to infinity.

(A4) $\mathcal{U} \in \mathcal{C}^{1}(\mathcal{D})$.

This smoothness assumption ensures that the velocity and the acceleration of the particle depend continuously on the position $r(t)$. If $\mathcal{D}=[0, h]$ we can relax this condition to $\mathcal{U} \in \mathcal{C}^{1}((0, h])$ so that we can allow potentials of the form $\mathcal{U}(r)=-\frac{1}{r}$ which are not defined at 0.

(A5) The density $g$ is spherically symmetric and satisfies

$$
g \in \mathcal{C}(\mathcal{D}) \cap \mathcal{C}^{1}\left(\mathcal{D}^{\circ}\right)
$$


together with

$$
\inf _{r \in \mathcal{D}} g(r)>0 .
$$

We require some smoothness from $g$ because the diffusion limit we get depends on the derivative $g^{\prime}$. The second assumption is needed because we do not want to have regions where the reflection rate goes to 0 . If one allows $g$ to approach 0 , then the free path may scale differently when the process is started in these regions. Examples of such situations are dealt with in Section 8 of [BR14].

1.4. Results. Our first result considers the asymptotic behavior of the steps of the free path Markov chain $\left(\left(\mathbf{X}_{k}^{n}, \mathcal{T}_{k}^{n}\right)\right)_{k \geq 0}$, where $\mathbf{X}_{k}^{n}$ is the location of the particle at the time of the $k$ th reflection and, anticipating our diffusion approximations, $n^{3 / 4} \mathcal{T}_{k}^{n}$ is the time of the $k$ th collision. This Markov chain has transition operator

$$
Q_{n} f(\mathbf{x}, t)=\mathbb{E}\left[f\left(\mathbf{y}\left[\mathbf{x}, v(\mathbf{x}) \mathbf{U}, N^{n}(\mathbf{x}, \mathbf{U})\right], t+n^{-3 / 4} N^{n}(\mathbf{x}, \mathbf{U})\right)\right] .
$$

The following result characterizes the asymptotic mean and covariance structure of the free path chain.

Theorem 1.3. Let

$$
\mu_{n}(\mathbf{x}, t):=n \mathbb{E}\left[\left(\mathbf{X}_{1}^{n}, \mathcal{T}_{1}^{n}\right)-(\mathbf{x}, t) \mid\left(\mathbf{X}_{0}^{n}, \mathcal{T}_{0}^{n}\right)=(\mathbf{x}, t)\right]
$$

be the scaled drift of $\left(\mathbf{X}_{k}^{n}, \mathcal{T}_{k}^{n}\right)_{k \geq 0}$, and let $S$ be a compact subset of $\mathcal{D}^{\circ}$. Then

$$
\lim _{n \rightarrow \infty} \sup _{(\mathbf{x}, t):\|\mathbf{x}\| \in S, t \geq 0}\left|\mu_{n}(\mathbf{x}, t)-\left(\frac{-1}{3 g(\mathbf{x})^{2}}\left[\frac{2 \nabla \mathcal{U}(\mathbf{x})}{m v(\mathbf{x})^{2}}+\frac{\nabla g(\mathbf{x})}{g(\mathbf{x})}\right], \frac{1}{g(\mathbf{x}) v(\mathbf{x})}\right)\right|=0,
$$

uniformly on $\{\mathbf{x}:\|\mathbf{x}\| \in S\} \times[0, \infty)$. Furthermore, let

$$
\begin{aligned}
\sigma_{n, i j}^{2}(\mathbf{x}, t) & :=n \mathbb{E}\left[\left(X_{1, i}^{n}-x_{i}\right)\left(X_{1, j}^{n}-x_{j}\right) \mid\left(\mathbf{X}_{0}^{n}, \mathcal{T}_{0}^{n}\right)=(\mathbf{x}, t)\right], \\
\sigma_{n, i t}^{2}(\mathbf{x}, t) & :=n \mathbb{E}\left[\left(X_{1, i}^{n}-x_{i}\right)\left(\mathcal{T}_{1}^{n}-t\right) \mid\left(\mathbf{X}_{0}^{n}, \mathcal{T}_{0}^{n}\right)=(\mathbf{x}, t)\right] \\
\sigma_{n, t}^{2}(\mathbf{x}, t) & :=n \mathbb{E}\left[\left(\mathcal{T}_{1}^{n}-t\right)^{2} \mid\left(\mathbf{X}_{0}^{n}, \mathcal{T}_{0}^{n}\right)=(\mathbf{x}, t)\right] .
\end{aligned}
$$

Then

(1) $\lim _{n \rightarrow \infty} \sup _{(\mathbf{x}, t):\|\mathbf{x}\| \in S, t \geq 0}\left|\sigma_{n, i j}^{2}(\mathbf{x}, t)-\frac{2}{3 g(\mathbf{x})^{2}} \delta_{i j}\right|=0$, where $\delta_{i j}=\mathbb{1}\{i=j\}$.

(2) $\lim _{n \rightarrow \infty} \sup _{(\mathbf{x}, t):\|\mathbf{x}\| \in S, t \geq 0} \sigma_{n, i t}^{2}(\mathbf{x}, t)=0$.

(3) $\lim _{n \rightarrow \infty} \sup _{(\mathbf{x}, t):\|\mathbf{x}\| \in S, t \geq 0} \sigma_{n, t}^{2}(\mathbf{x}, t)=0$.

This theorem is a combination of Lemmas 2.10 and 2.11 below. Heuristically, this says that both $\mathcal{U}_{n}$ and $g_{n}$ impart a drift towards areas where the corresponding function has a smaller value. That is, the particle prefers to move towards areas where it travels quickly and where there are few scatterers, which may be competing influences. It is interesting to note that the potential $\mathcal{U}$ does not appear in the covariance terms. It can, and will, effect the diffusion coefficient of the limiting diffusion only through an overall time change.

With Theorem 1.3 in hand, it is straightforward to obtain the following diffusion approximation, which separates out the effects of the particle's position at times of reflection from the effects of speed at which the particle is moving. Let $\mathcal{D}_{3}=\{\mathbf{x} \in$ $\left.\mathbb{R}^{3}:\|\mathbf{x}\| \in \mathcal{D}\right\}$. 
Theorem 1.4. Let $\left(\mathcal{X}_{t}\right)_{t \geq 0}$ be a diffusion on $\mathcal{D}_{3}$ whose generator $G$ acts on functions $f \in C^{2}\left(\mathcal{D}_{3}\right)$ with compact support in $\mathcal{D}_{3}^{\circ}$ by

$$
G f(\mathbf{x})=\frac{1}{3 g^{2}(\mathbf{x})} \Delta f(\mathbf{x})-\frac{1}{3 g^{2}(\mathbf{x})}\left(\frac{\nabla g(\mathbf{x})}{g(\mathbf{x})}+\frac{2 \nabla \mathcal{U}(\mathbf{x})}{m v(\mathbf{x})^{2}}\right) \cdot \nabla f(\mathbf{x})
$$

and killed if $/$ when $\mathcal{R}:=\|\mathcal{X}\|$ hits the boundary of $\mathcal{D}$. Consider any $l, u \in \mathcal{D}^{\circ}$ with $l<u$ and start the process $\left(\left(\mathbf{X}_{k}^{n}, \mathcal{T}_{k}^{n}\right), k \in \mathbb{N}_{0}\right)$ at $(\mathbf{x}, 0)$, where $l<\|\mathbf{x}\|<u$. Define the stopping times

$$
\tau_{l, u}^{n}:=\inf \left\{k \in \mathbb{N}_{0}:\left\|\mathbf{X}_{k}^{n}\right\| \notin[l, u]\right\}
$$

and

$$
\tau_{l, u}:=\inf \left\{t \geq 0:\left\|\mathcal{X}_{t}\right\| \notin[l, u]\right\} .
$$

Then, as $n \rightarrow \infty$, the family of continuous time processes $\left(\left(\mathbf{X}_{\lfloor n t\rfloor \wedge \tau_{l, u}^{n}}^{n}, \mathcal{T}_{\lfloor n t\rfloor \wedge \tau_{l, u}^{n}}^{n}\right)\right.$, $t \geq 0)$ converges in distribution on the Skorokhod space $D\left(\mathbb{R}_{+}, \mathbb{R}\right)$ to the diffusion

$$
\left(\left(\mathcal{X}_{t \wedge \tau_{l, u}}, \int_{0}^{t \wedge \tau_{l, u}} \frac{d s}{g\left(\mathcal{X}_{s}\right) v\left(\mathcal{X}_{s}\right)}\right), t \geq 0\right)
$$

with initial position $(\mathbf{x}, 0)$. This convergence happens jointly with the convergence of the hitting times, $n^{-1} \tau_{l, u}^{n} \Rightarrow \tau_{l, u}$.

This theorem is proved in Section 3, where it is restated for convenience.

Remark 1.5. The convergence above also holds without stopping near a boundary point of $\mathcal{D}$ that is inaccessible for the diffusion $\mathcal{R}=\|\mathcal{X}\|$. See Section 5 for how one can determine when a point is inaccessible. In particular, the boundaries are inaccessible for the constant acceleration towards the origin and for Newtonian gravity centered at the origin.

Remark 1.6. This type of limit theorem, that gives joint convergence of a process observed when it changes direction and the time spent between such changes up to that point, is typical of the continuous time random walk literature; see e.g. [MS14c]. Although our models can naturally be viewed as transport processes, our results on the free path of the particle suggest an approach to diffusive limits that has more in common with that used to study Continuous Time Random Walks (CTRW) MS04, MS14c than that typically used to study transport processes. CTRW models also arise in many physical applications (see e.g. [ $\mathrm{WZL}^{+}$14, MK00, BCDS06, SBMB03]) and have been particularly useful in studying anomalous diffusions. The advantage of this approach is that, on the diffusive scale, we may easily distinguish between the effects of the particle's displacement between collisions and the effects of the speed at which the particle is traveling. In this way, our approach is similar to the one taken in [BR14, and our work can be seen as an extension of the methods used there. This approach is also similar to the approach in CD09 to studying the two-dimensional periodic Lorentz gas in a gravitational field, though, of course, our setting is far simpler.

By inverting the time process $\left(\mathcal{T}_{k}^{n}\right)_{k \geq 0}$ and showing that the free path is typically not far from the straight line between its endpoints, we can arrive at a diffusion approximation for $\left(\mathbf{X}^{n}(t)\right)_{t \geq 0}$, the trajectory of the particle. In particular, we obtain the following result. 
Theorem 1.7. Let $\left(\mathbf{X}^{n}(t)\right)$ denote the trajectory of the particle and fix $l, u \in \mathcal{D}^{\circ}$ with $l<u$. Suppose that $\left\|\mathbf{X}^{n}(0)\right\| \in(l, u)$ and define

$$
\iota_{l, u}^{n}:=\inf \left\{t:\left\|\mathbf{X}^{n}(t)\right\| \notin[l, u]\right\} .
$$

We have the following convergence in distribution on the Skorokhod space $D\left(\mathbb{R}_{+}, \mathbb{R}\right)$ :

$$
\left(\mathbf{X}^{n}\left(\left(n^{3 / 4} t\right) \wedge \iota_{l, u}^{n}\right)\right)_{t \geq 0} \rightarrow\left(\mathcal{X}\left(\Omega(t) \wedge \tau_{l, u}\right)\right)_{t \geq 0},
$$

where $\mathcal{X}$ is as in Theorem 3.1 and $\Omega$ is the time change given by

$$
\Omega(t):=\mathcal{I}\left(\int_{0}^{\cdot} \frac{d s}{g\left(\mathcal{X}\left(s \wedge \tau_{l, u}\right)\right) v\left(\mathcal{X}\left(s \wedge \tau_{l, u}\right)\right)}\right)(t)
$$

and $\mathcal{I}$ is the inverse operator defined by $\mathcal{I}(f)(t)=\inf \{s: f(s)>t\}$. Furthermore, the time change process $(\mathcal{X}(\Omega(t)), t \geq 0)$ is a diffusion process whose generator $\mathscr{G}$ acts on functions $f \in C^{2}\left(\mathcal{D}_{3}\right)$ with compact support in $\mathcal{D}_{3}^{\circ}$ by

$$
\mathscr{G} f(\mathbf{x})=g(\mathbf{x}) v(\mathbf{x}) G f(\mathbf{x})=\frac{v(\mathbf{x})}{3 g(\mathbf{x})} \Delta f(\mathbf{x})-\frac{v(\mathbf{x})}{3 g(\mathbf{x})}\left(\frac{\nabla g(\mathbf{x})}{g(\mathbf{x})}+\frac{2 \nabla \mathcal{U}(\mathbf{x})}{m v(\mathbf{x})^{2}}\right) \cdot \nabla f(\mathbf{x}) .
$$

This theorem is proved in Section 4, where it is restated for convenience.

Remark 1.8. The stopping at $u$ and/or at $l$ can be removed when the left and/or right boundary points of $\mathcal{D}$ are inaccessible for the diffusion $\mathcal{R}$. See Remark 1.5 and Section 5 .

Remark 1.9. The cutoffs are necessary because of the generality the potentials and scattering densities we allow permit very different behaviors at the boundary. In some cases we expect the boundaries to be inaccessible (see Section 5), while in other cases we expect the boundaries to be reflecting as in RT99 and in yet others, like $\mathcal{U}(r)=-r^{-2}$, the origin should trap the particle. We leave a detailed investigation of the boundary as an open problem.

Remark 1.10. Our results have much in common with Cos91, but there are key differences at both heuristic and technical levels and, as a result, there is no overlap between the precise results. For instance, our models do not satisfy the underlying technical assumptions of Cos91. The function $Q^{-1}$ from Cos91, which controls the reflection rate, has to be differentiable up to the boundary of the region where the motion takes place (see assumption (H2) from [Cos91]). In our case we have singularities at the origin and when $\mathcal{D}=[0, h]$ we also have singularities at the boundary of the domain. Furthermore, the speed of the particle must be bounded away from 0 and $\infty$ in Cos91, while there is no such restriction in our model. This is related to the fact that Cos91 restricts the particle's motion by imposing a reflecting boundary while in our case the domain of the particle's motion is completely determined by $\mathcal{U}$ and $E$.

Remark 1.11. The form of the generator (1.1) puts the random flight process in the class of processes known as transport processes. There is a substantial literature on diffusion approximations of transport processes and, more generally, random evolutions. Very general approaches and results can be found, for example, in Kur73, Pap75, BLP79, Cos91. In order to apply these general techniques the generator needs to be smoothed to control for regions where the particle's velocity is low. Nonetheless, it is possible to adapt these approaches to obtain Theorem 1.7 
However, our results on the free path are stronger than the diffusion approximation results and cannot be recovered by these general methods. Moreover, these methods do not clarify the connection with the free path chain.

\section{The FREE PATH of THE PARTICLE}

In this section we analyze the jumps of the free flight chain $\left(\left(\mathbf{X}_{k}^{n}, \mathcal{T}_{k}^{n}\right)\right)_{k \geq 0}$ whose transition operator is given by (1.16). Our proofs are complicated by the fact that for a general potential $\mathcal{U}$ it is not possible to explicitly find the trajectory of the particle in the absence of collisions and this makes it difficult to determine how long this path spends in domains where it is traveling slowly. Our first lemma will give us local control over how the radial part of the path behaves.

Lemma 2.1. For any $r_{0} \in \mathcal{D}^{\circ}$ and $\theta \in[-\pi, \pi]$

$$
r(t):=r\left(r_{0}, \theta, t\right)=r_{0}+v_{0} \cos (\theta) \cdot t+\frac{1}{2}\left(\frac{-\partial_{r} \mathcal{U}(r(\tau))}{m}+\frac{v_{0}^{2} r_{0}^{2} \sin ^{2}(\theta)}{r(\tau)^{3}}\right) t^{2}
$$

for some $0 \leq \tau \leq t$ depending on $r_{0}$, $\theta$, and $t$.

Proof. By definition $\mathbf{r}(t):=\left(r\left(r_{0}, \theta, t\right), \alpha\left(r_{0}, \alpha_{0}, \theta, t\right)\right)$ is the solution to the equations of motion in polar coordinates for a particle of mass $m$ in the potential $\mathcal{U}$. Equation (1.9) implies

$$
\frac{1}{r} \frac{d}{d t}\left(r^{2} \dot{\alpha}\right)=0
$$

and

$$
\ddot{r}-r \dot{\alpha}^{2}=-\frac{\partial_{r} \mathcal{U}(r)}{m} .
$$

As a result of (2.1) and (1.10)

$$
\dot{\alpha}(t)=\frac{v\left(r_{0}\right) r_{0} \sin \theta}{r(t)^{2}} .
$$

By (2.2) and (2.3)

$$
\ddot{r}(t)=\frac{-\partial_{r} \mathcal{U}(r(t))}{m}+\frac{v\left(r_{0}\right)^{2} r_{0}^{2} \sin ^{2}(\theta)}{r(t)^{3}} .
$$

Taylor expanding $r(t)$ and using (2.2), (1.10) together with (2.3) yields

$$
r(t)=r_{0}+v\left(r_{0}\right) \cos (\theta) \cdot t+\frac{1}{2}\left(\frac{-\partial_{r} \mathcal{U}(r(\tau))}{m}+\frac{v\left(r_{0}\right)^{2} r_{0}^{2} \sin ^{2}(\theta)}{r(\tau)^{3}}\right) t^{2} .
$$

Remark 2.2. Throughout the remainder of this section we let $S, S^{\prime}$ be closed intervals satisfying

$$
S \subset\left(S^{\prime}\right)^{\circ} \subset S^{\prime} \subset \mathcal{D}^{\circ} .
$$

Also, for any $\delta>0$, we define

$$
\Lambda_{\delta}^{n}(S)=\inf _{(\mathbf{x}, \mathbf{u}):\|\mathbf{x}\| \in S, \mathbf{u} \in \mathbf{S}^{2}} \inf _{t \geq 0}\left\{t:\left\|\mathbf{y}_{n}(\mathbf{x}, \mathbf{u}, t)-\mathbf{x}\right\| \geq \delta\right\}
$$

to be the shortest time it takes for the particle's displacement from its initial position to be at least $\delta$ when started inside $S$.

If

$$
0<\delta<d\left(S,\left(S^{\prime}\right)^{c}\right):=\inf \left\{|x-y|: x \in S, y \in \mathcal{D} \backslash S^{\prime}\right\}
$$


then for all $(\mathbf{x}, \mathbf{u}, t) \in\{\mathbf{x}:\|\mathbf{x}\| \in S\} \times \mathbf{S}^{2} \times\left[0, \Lambda_{\delta}^{n}(S)\right]$ one has

$$
\left\|\mathbf{y}_{n}(\mathbf{x}, \mathbf{u}, t)\right\| \in S^{\prime}
$$

Since $\left\|\dot{\mathbf{y}}_{n}\right\|=n^{-1 / 4} v(\mathbf{y})$, we have

$$
\Lambda_{\delta}^{n}(S) \geq n^{1 / 4} \cdot \frac{\delta}{\sup _{r_{0} \in S^{\prime}} v\left(r_{0}\right)}>0
$$

where $\sup _{r_{0} \in S^{\prime}} v\left(r_{0}\right)<\infty$ since $S^{\prime}$ is bounded away from $\partial D$.

The next lemma shows that the scaled trajectories converge to the starting point, uniformly over a fixed time interval.

Lemma 2.3. Fix $T>0$. Then

$$
\begin{array}{r}
\lim _{n \rightarrow \infty} \sup _{(\mathbf{x}, \mathbf{u}, t) \in\{\mathbf{x}:\|\mathbf{x}\| \in S\} \times \mathbf{S}^{2} \times[0, T]}\left\|\mathbf{y}_{n}(\mathbf{x}, \mathbf{u}, t)-\mathbf{x}\right\|=0, \\
\lim _{n \rightarrow \infty} \sup _{(\mathbf{x}, \mathbf{u}, t) \in\{\mathbf{x}:\|\mathbf{x}\| \in S\} \times \mathbf{S}^{2} \times[0, T]}\left\|\sqrt{n} \ddot{\mathbf{y}}_{n}(\mathbf{x}, \mathbf{u}, t)-\frac{\nabla \mathcal{U}(\mathbf{x})}{m}\right\|=0, \\
\lim _{n \rightarrow \infty} \sup _{\left(r_{0}, \theta, t\right) \in S \times[-\pi, \pi] \times[0, T]}\left|r_{n}\left(r_{0}, \theta, t\right)-r_{0}\right|=0,
\end{array}
$$

and

$$
\lim _{n \rightarrow \infty} \sup _{\left(r_{0}, \theta, t\right) \in S \times[-\pi, \pi] \times[0, T]}\left|\sqrt{n} \ddot{r}_{n}\left(r_{0}, \theta, t\right)-\left(-\frac{\partial_{r} \mathcal{U}\left(r_{0}\right)}{m}+\frac{v^{2}\left(r_{0}\right) \sin ^{2}(\theta)}{r_{0}}\right)\right|=0 .
$$

Proof. The claims about $r_{n}$ are slightly more complicated, so we prove those and leave the more obvious claims about $\mathbf{y}_{n}$ to the reader. Let $\delta>0$ be such that $\delta \leq$ $d\left(S,\left(S^{\prime}\right)^{c}\right)$. By Remark 2.2 there exists $M \in \mathbb{N}$ large enough such that $\Lambda_{\delta}^{n}(S) \geq T$ whenever $n \geq M$. Equivalently, for $n \geq M$ we have

$$
\left|r_{n}\left(r_{0}, \theta, t\right)-r_{0}\right| \leq \delta
$$

for all $\left(r_{0}, \theta, t\right) \in S \times[-\pi, \pi] \times[0, T]$. This proves the uniform convergence of $r_{n}\left(r_{0}, \theta, t\right)$ to $r_{0}$. Define

$$
\psi\left(r_{0}, r_{0}, \theta\right):=-\frac{\partial_{r} \mathcal{U}\left(r_{0}\right)}{m}+\frac{v^{2}\left(r_{0}\right) r_{0}^{2} \sin ^{2}(\theta)}{r_{0}^{3}}
$$

and note by (2.4) that

$$
\ddot{r}_{n}\left(r_{0}, \theta, t\right)=\frac{1}{\sqrt{n}} \psi\left(r_{n}(t), r_{0}, \theta\right) .
$$

$S^{\prime}$ is bounded away from 0 and $\mathcal{U} \in \mathcal{C}^{1}\left(\mathcal{D}^{\circ}\right)$ imply that $\psi$ is uniformly continuous on $S^{\prime} \times S \times[-\pi, \pi]$. By construction $r_{n}(t) \in S^{\prime}$ for all $n \geq M$ and $t \in[0, T]$. Because $r_{n}\left(r_{0}, \theta, t\right)$ converges uniformly to $r_{0}$ on compact sets, we have

$$
\lim _{n \rightarrow \infty} \sup _{\left(r_{0}, \theta, t\right) \in S \times[-\pi, \pi] \times[0, T]}\left\{\left|\sqrt{n} \ddot{r}_{n}\left(r_{0}, \theta, t\right)-\psi\left(r_{0}, r_{0}, \theta\right)\right|\right\}=0,
$$

where

$$
\psi\left(r_{0}, r_{0}, \theta\right)=-\frac{\partial_{r} \mathcal{U}\left(r_{0}\right)}{m}+\frac{v^{2}\left(r_{0}\right) \sin ^{2}(\theta)}{r_{0}}
$$

by (2.8). This completes the proof. 
Since the free flight chain tracks the process at reflection times, we want to apply the estimates for (2.5) between reflections. By (1.3) and the rescaling, we know that for every $k$, if at $T_{k}$ the particle is at position $\left(r_{0}, \alpha_{0}\right)$ and reflects in the direction given by the angle $\theta$, then $T_{k+1}^{n}-T_{k}^{n}$ is distributed like the random variable $N^{(n)}\left(r_{0}, \theta\right)$ which we define by

$$
\mathbb{P}\left(N^{(n)}\left(r_{0}, \theta\right)>t\right)=\exp \left(-\int_{0}^{t} n^{1 / 4} \lambda\left(r_{n}\left(r_{0}, \theta, s\right)\right) d s\right),
$$

where

$$
\lambda\left(r_{0}\right):=g\left(r_{0}\right) v\left(r_{0}\right) .
$$

Throughout, we will often suppress the $r_{0}$ and $\theta$ dependencies of $N^{(n)}\left(r_{0}, \theta\right)$ and write $N^{(n)}$ or $N^{(n)}(\theta)$ when no confusion will arise. To go back and forth between Euclidean and polar coordinates, note that we have the identity in distribution

$$
N^{(n)}(\mathbf{x}, \mathbf{u})={ }_{d} N^{(n)}\left(\|\mathbf{x}\|, \cos ^{-1}\left(\frac{\mathbf{u} \cdot \mathbf{x}}{\|\mathbf{x}\|}\right)\right),
$$

where $N^{(n)}(\mathbf{x}, \mathbf{u})$ is defined as in (1.8) with the appropriately rescaled parameters. Consequently, for studying the asymptotic duration of the free path, there is no loss in studying $N^{(n)}(r, \theta)$ in a uniform way over $r$ and $\theta$.

For many of our proofs we require estimates that show the time between reflections approaches 0 with high probability as the scaling factor $n$ goes to infinity. This will allow us to apply the local estimates from the expansion of $r(t)$ in Lemma 2.1 and to show the free flight process does not undergo large jumps. We first prove some bounds on the moments of $N^{(n)}\left(r_{0}, \theta\right)$.

Lemma 2.4. The family

$$
\left\{n^{1 / 4} N^{(n)}\left(r_{0}, \theta\right):\left(r_{0}, \theta, n\right) \in S \times[-\pi, \pi] \times \mathbb{N}\right\}
$$

is bounded in $L^{p}$ for $1 \leq p<\infty$.

Proof. We will assume that $\mathcal{D}=[0, h]$ or $\mathcal{D}=[0, \infty)$. The cases $\mathcal{D}=\left[h_{-}, h_{+}\right]$and $[h, \infty)$ can be treated similarly.

Case I $(\mathcal{D}=[0, h])$. By Assumptions (A3) and (A4) there exist $\delta>0$ and $U_{\delta}>0$ such that

$$
\min _{[h-\delta, h]}\left|\partial_{r} U\left(r_{0}\right)\right| \geq U_{\delta} .
$$

Let $S \subset D^{\circ}$ be a compact set, let $\eta>0$, and assume our particle enters the annulus $A_{\eta}$ with inner radius $h-\eta$ and outer radius $h$ at time $t_{0}$. By (1.12)

$$
v(h-\eta)=\sqrt{\frac{2}{m}(E-\mathcal{U}(h-\eta))} .
$$

Using (2.2) and (2.3)

$$
\ddot{r}(t)=-\frac{\partial_{r} \mathcal{U}(r(t))}{m}+r(t) \dot{\alpha}^{2}(t) \leq-\frac{\partial_{r} \mathcal{U}(r(t))}{m}+\frac{[v(h-\eta)]^{2}}{r} .
$$

Let

$$
t_{e}:=\inf \left\{s>t_{0}: r(s)=h-\eta\right\}
$$

be the time when the particle exits the annulus. By Assumption (A3)

$$
\mathcal{U}(h)=E .
$$


Since $\mathcal{U}$ is continuous this means that we can make $v(r)$ as small as we like if we are close enough to $r=h$. This together with (2.12), (2.13), and (2.14) implies that there exist $\gamma>0, m_{\gamma}>0$ such that

$$
\ddot{r}(t) \leq-m_{\gamma}
$$

whenever $r(t) \in[h-\gamma, h]$. Set $\eta=\gamma$. Note that $t_{e}-t_{0}$ is bounded above by the time it would take a particle started at $r=h-\gamma$ with speed $\dot{r}\left(t_{0}\right)>0$ pointed along the radius and with acceleration $\ddot{r}=-m_{\gamma}<0$ to return to $r=h-\gamma$. Thus,

$$
t_{e}-t_{0} \leq 2 \frac{\dot{r}\left(t_{0}\right)}{m_{\gamma}}<\infty .
$$

Next, define

$$
t_{r}:=\inf \left\{s>t_{e}: r(s)=h-\gamma\right\} .
$$

This is the first return time to the annulus $A_{\gamma}$. We want to bound $t_{r}-t_{e}$ below. If $\dot{r}\left(t_{0}\right) \leq 0$, then the particle would not spend any time in the annulus $A_{\gamma}$, that is, $t_{e}-t_{0}=0$. Therefore, we can assume that $\dot{r}\left(t_{0}\right)>0$. By conservation of angular momentum and conservation of energy we have that $v\left(t_{0}\right)=v\left(t_{e}\right)$ and $\dot{\alpha}\left(t_{e}\right)=\dot{\alpha}\left(t_{0}\right)$. This implies that $\dot{r}\left(t_{e}\right)=-\dot{r}\left(t_{0}\right)<0$. Since $\ddot{r}$ is finite on $S$ we immediately get that

$$
\begin{aligned}
t_{r}-t_{e} & \geq \frac{2 \dot{r}\left(t_{0}\right)}{\sup _{S}|\ddot{r}|} \geq \frac{2 \dot{r}\left(t_{0}\right)}{\frac{\sup _{S}\left|\partial_{r} \mathcal{U}(r)\right|}{m}+\sup _{S}\left|r \dot{\alpha}^{2}\right|} \\
& \geq \frac{2 \dot{r}\left(t_{0}\right)}{\frac{\sup _{S}\left|\partial_{r} \mathcal{U}(r)\right|}{m}+\sup _{S} \frac{v^{2}(r)}{r}}>0 .
\end{aligned}
$$

Combining (2.15) and (2.16),

$$
\begin{aligned}
\sup _{S \times[-\pi, \pi]} \frac{t_{e}-t_{0}}{t_{r}-t_{e}} & =\sup _{S \times[-\pi, \pi]} \frac{2 \frac{\dot{r}\left(t_{0}\right)}{m_{\gamma}}}{\frac{2 \dot{r}\left(t_{0}\right)}{\frac{\sup _{S}\left|\partial_{r} \mathcal{U}(r)\right|}{m}+\sup _{S} \frac{v^{2}(r)}{r}}} \\
& \leq \frac{\frac{\sup _{S}\left|\partial_{r} \mathcal{U}(r)\right|}{m}+\sup _{S} \frac{v^{2}(r)}{r}}{m_{\gamma}}<\infty,
\end{aligned}
$$

where we assume that if $t_{0}=\infty$, then $t_{e}-t_{0}=0$ and

$$
\frac{t_{e}-t_{0}}{t_{r}-t_{e}}=0 \text {. }
$$

Note that the upper bound from (2.17) is the same if we use the rescaled trajectory of the particle $\left(r_{n}(t), \alpha_{n}(t)\right)$. This follows from the way we found $m_{\lambda}$ and by Assumption (A1).

By Assumption (A2) we know that

$$
\inf _{S} v\left(r_{0}\right)>0
$$

Suppose that $t \geq \sup _{S}\left(t_{e}-t_{0}\right)+\inf _{S}\left(t_{r}-t_{e}\right)$. Using (2.17) together with the fact that the worst case scenario is when the particle spends the longest possible time in the 'bad region' $A_{\gamma}$ and the least amount of time in the 'good region' $\mathcal{D} \backslash A_{\gamma}$, 
we have for some $c>0$ that

$$
\begin{aligned}
\mathbb{P}\left\{N^{(n)}>t\right\} & =\exp \left(-\int_{0}^{t} n^{1 / 4} g\left(r_{n}\right) v\left(r_{n}\right) d s\right) \\
& \leq \exp \left(-\int_{0}^{\left(\frac{1}{1+c}\right) t} n^{1 / 4} \inf _{S} g(r) \inf _{S} v(r) d s\right) \\
& =\exp \left(-\frac{1}{1+c} t n^{1 / 4} \inf _{S} g(r) \inf _{S} v(r)\right)
\end{aligned}
$$

which decays exponentially in $n$ as $n \rightarrow \infty$ as long as $t$ is large. Therefore,

$$
\begin{aligned}
\mathbb{E}\left[\left(n^{1 / 4} N^{(n)}\right)^{p}\right] & =\int_{0}^{\infty} p t^{p-1} \mathbb{P}\left(n^{1 / 4} N^{(n)}>t\right) d t \\
& \leq \int_{0}^{\infty} p t^{p-1} \exp \left(-\frac{1}{1+c} t \inf _{S} g(r) \inf _{S} v(r)\right) d t \\
& <\infty .
\end{aligned}
$$

Since the bound above does not depend on $r, \theta$, or $n$ we get that

$$
\sup _{S \times[-\pi, \pi] \times \mathbb{N}} \mathbb{E}\left[\left(n^{1 / 4} N^{(n)}\left(r_{0}, \theta\right)\right)^{p}\right]<\infty .
$$

Case II $(\mathcal{D}=[0, \infty))$. Set $g_{\text {min }}:=\inf _{r \in \mathcal{D}} g(r)$. We know by Assumption (A3) that there exists $\bar{\delta}>0$ such that $\inf _{\mathcal{D}} v(r) \geq \bar{\delta}$ so by (2.10)

$$
\mathbb{P}\left\{N^{(n)}>t\right\} \leq \exp \left(-n^{1 / 4} \bar{\delta} t g_{\min }\right)
$$

which, like before, forces

$$
\begin{aligned}
\mathbb{E}\left[\left(n^{1 / 4} N^{(n)}\right)^{p}\right] & =\int_{0}^{\infty} p t^{p-1} \mathbb{P}\left(n^{1 / 4} N^{(n)}>t\right) d t \\
& \leq \int_{0}^{\infty} p t^{p-1} \exp \left(-\bar{\delta} t g_{\min }\right) d t \\
& <\infty
\end{aligned}
$$

Similarly to Case I, because the bound above does not depend on $r, \theta$, or $n$ we get that

$$
\sup _{S \times[-\pi, \pi] \times \mathbb{N}} \mathbb{E}\left[\left(n^{1 / 4} N^{(n)}\left(r_{0}, \theta\right)\right)^{p}\right]<\infty .
$$

Lemma 2.4 also provides us with the following corollary which will prove useful in showing that the probability that $N^{(n)}\left(r_{0}, \theta\right)$ is larger than any fixed value decays rapidly as the scaling parameter $n$ goes to infinity.

Corollary 2.5. For all $k \in \mathbb{R}$ and for all $\varepsilon>0$,

$$
\lim _{n \rightarrow \infty} \sup _{\left(r_{0}, \theta\right) \in S \times[-\pi, \pi]} n^{k} \mathbb{P}\left\{N^{(n)}\left(r_{0}, \theta\right) \geq \varepsilon\right\}=0 .
$$

Proof. This is immediate from Lemma 2.4.

In addition, we also have the following corollary that shows that tails of the moments of $N^{(n)}\left(r_{0}, \theta\right)$ decay rapidly as well. 
Corollary 2.6. For all $k \in \mathbb{R}, l \geq 1$, and $\varepsilon>0$

$$
\lim _{n \rightarrow \infty} \sup _{\left(r_{0}, \theta\right) \in S \times[-\pi, \pi]} n^{k} \mathbb{E}\left[\left(N^{n}\left(r_{0}, \theta\right)\right)^{l} \mathbb{1}_{\left\{N^{(n)}>\varepsilon\right\}}\right]=0 .
$$

Proof. Cauchy-Schwarz together with Lemma 2.4 and Corollary 2.5 yield the desired result.

From Lemma 2.4, we have the following estimates on the moments of $N^{(n)}$.

Lemma 2.7. Let $1 \leq p<\infty$. Then

$\lim _{n \rightarrow \infty} \sup _{\left(r_{0}, \theta\right) \in S \times[-\pi, \pi]}\left|\mathbb{E}\left(n^{p / 4}\left[N^{(n)}\left(r_{0}, \theta\right)\right]^{p}\right)-\int_{0}^{\infty} p t^{p-1} \exp \left(-g\left(r_{0}\right) v\left(r_{0}\right) t\right) d t\right|=0$.

Proof. First let $M \in \mathbb{R}_{+}$and note that the truncated moments $\mathbb{E}\left[n^{p / 4}\left[N^{(n)}\left(r_{0}, \theta\right)\right]^{p}\right.$ $\left.\wedge M^{p}\right]$ can be written as

$$
\begin{aligned}
\mathbb{E}\left[n^{p / 4}\left[N^{(n)}\left(r_{0}, \theta\right)\right]^{p} \wedge M^{p}\right] & =p \int_{0}^{\infty} t^{p-1} \mathbb{P}\left[n^{1 / 4} N^{(n)}\left(r_{0}, \theta\right) \wedge M>t\right] d t \\
& =p \int_{0}^{M} t^{p-1} \mathbb{P}\left[n^{1 / 4} N^{(n)}\left(r_{0}, \theta\right)>t\right] d t .
\end{aligned}
$$

Making the change of variables $u=n^{1 / 4} s$, we have

$$
\mathbb{P}\left(n^{1 / 4} N^{(n)}\left(r_{0}, \theta\right)>t\right)=\exp \left(-\int_{0}^{t} g\left(r\left(r_{0}, \theta, u / n^{1 / 4}\right)\right) v\left(r_{0}, \theta, u / n^{1 / 4}\right)\right) d u .
$$

Both $r=r\left(r_{0}, \theta, t\right)$ and $v=v\left(r_{0}, \theta, t\right)$ are continuous functions on $\mathcal{O}:=S \times$ $[-\pi, \pi] \times[0, M]$. Since $\mathcal{O}$ is compact, $r$ and $v$ are in fact uniformly continuous on $S$. By Assumption (A5) $g$ is continuous on $S$, and therefore is uniformly continuous. This implies that $g \circ r$ is uniformly continuous on $\mathcal{O}$. By Lemma 2.3 it follows that

$$
\lim _{n \rightarrow \infty} g\left(r_{n}\left(r_{0}, \theta, u / n^{1 / 4}\right)\right)=g\left(r\left(r_{0}, \theta, 0\right)\right)=g\left(r_{0}\right)
$$

and

$$
\lim _{n \rightarrow \infty} v\left(r_{0}, \theta, u / n^{1 / 4}\right)=v\left(r_{0}, \theta, 0\right):=v\left(r_{0}\right)
$$

both uniformly on $\mathcal{O}$. As a result, $\mathbb{P}\left(n^{1 / 4} N^{(n)}\left(r_{0}, \theta\right)>t\right)$ converges uniformly to $\exp \left(-g\left(r_{0}\right) v\left(r_{0}\right) t\right)$ on $\mathcal{O}$, which implies

$$
\begin{array}{r}
\lim _{n \rightarrow \infty} \sup _{\left(r_{0}, \theta\right) \in S \times[-\pi, \pi]} \mid \mathbb{E}\left[n^{p / 4} N^{(n)}\left(r_{0}, \theta\right)^{p} \wedge M^{p}\right] \\
-\int_{0}^{M} p t^{p-1} \exp \left(-g\left(r_{0}\right) v\left(r_{0}\right) t\right) \mid=0 .
\end{array}
$$

It is then standard to extend the result to the expectation without the truncation.

For the cases $p=1,2$ we have by Lemma 2.7

$$
\lim _{n \rightarrow \infty} \sup _{\left(r_{0}, \theta\right) \in S \times[-\pi, \pi]}\left|\mathbb{E}\left[n^{1 / 4} N^{(n)}\left(r_{0}, \theta\right)\right]-\frac{1}{g\left(r_{0}\right) v\left(r_{0}\right)}\right|=0
$$


and

$$
\lim _{n \rightarrow \infty} \sup _{\left(r_{0}, \theta\right) \in S \times[-\pi, \pi]}\left|\mathbb{E}\left[n^{1 / 2}\left(N^{(n)}\left(r_{0}, \theta\right)\right)^{2}\right]-\frac{2}{g^{2}\left(r_{0}\right) v^{2}\left(r_{0}\right)}\right|=0 .
$$

The next result shows that on the event $\left\{N^{(n)}>\varepsilon\right\}$ the $k$ th moment of the displacement of the particle at time 0 and at the first reflection $N^{(n)}$ decays faster than $\frac{1}{n^{m}}$ as $n \rightarrow \infty$.

Lemma 2.8. Fix $\varepsilon>0, m, k \in \mathbb{N}$. Then

$$
\lim _{n \rightarrow \infty} \sup _{\left\{\left(\mathbf{x}_{0}, \mathbf{u}\right):\left\|\mathbf{x}_{0}\right\| \in S, \mathbf{u} \in \mathbf{S}^{2}\right\}} n^{m} \mathbb{E}\left[\left\|\mathbf{y}_{n}\left(N^{(n)}(\mathbf{u})\right)-\mathbf{x}_{0}\right\|^{k} \mathbb{1}_{\left\{N^{(n)}>\varepsilon\right\}}\right]=0 .
$$

Proof. Since $\left\|\mathbf{y}^{\prime}\right\|=v_{n}(\mathbf{y})$ we have

$$
\begin{array}{r}
\mathbb{E}\left[\left\|\mathbf{y}_{n}\left(N^{(n)}(\mathbf{u})\right)-\mathbf{x}_{0}\right\|^{k} \mathbb{1}_{\left\{N^{(n)}>\varepsilon\right\}}\right] \leq \mathbb{E}\left[\left(\int_{0}^{N^{(n)}} v_{n}(\mathbf{y}(s)) d s\right)^{k} \mathbb{1}_{\left\{N^{(n)}>\varepsilon\right\}}\right] \\
\leq n^{-k / 2} g_{\min }^{-k}\left(\mathbb{E}\left[\left(\int_{0}^{N^{(n)}} g_{n}(\mathbf{y}(s)) v_{n}(\mathbf{y}(s)) d s\right)^{2 k}\right]\right)^{1 / 2} \mathbb{P}\left(N^{(n)}>\varepsilon\right)^{1 / 2} .
\end{array}
$$

The lemma now follows from Corollary 2.5 and the fact that

$$
\int_{0}^{N^{(n)}} g_{n}(\mathbf{y}(s)) v_{n}(\mathbf{y}(s)) d s
$$

is distributed like an $\operatorname{Exp}(1)$ random variable and, consequently, has finite moments.

Lemma 2.9. Let $\mathbf{U}$ be uniformly distributed on $\mathbf{S}^{2}$. Then

$$
\lim _{n \rightarrow \infty} \sup _{\mathbf{x}:\|\mathbf{x}\| \in S}\left\|n^{3 / 4} \mathbb{E}\left[N^{(n)}(\mathbf{x}, \mathbf{U}) \mathbf{U}\right]-\frac{1}{3 g(\mathbf{x})^{2} v(\mathbf{x})}\left(\frac{\nabla \mathcal{U}(\mathbf{x})}{m v(\mathbf{x})^{2}}-\frac{\nabla g(\mathbf{x})}{g(\mathbf{x})}\right)\right\|=0 .
$$

Proof. First, for notational convenience, define the auxiliary function

$$
\begin{aligned}
F_{n}(\mathbf{x}, \mathbf{u}, t) & :=\int_{0}^{t} g_{n}\left(\mathbf{y}_{n}(\mathbf{x}, \mathbf{u}, s)\right) v_{n}\left(\mathbf{y}_{n}(\mathbf{x}, \mathbf{u}, s)\right) d s \\
& =\int_{0}^{t} g_{n}\left(r_{n}(\|\mathbf{x}\|, \theta, s)\right) v_{n}\left(r_{n}(\|\mathbf{x}\|, \theta, s)\right) d s
\end{aligned}
$$

where $\theta=\cos ^{-1}(\mathbf{u} \cdot \mathbf{x} /\|\mathbf{x}\|)$. In the usual way, we will often suppress the $\mathbf{x}$ and $\mathbf{u}$ dependencies of $F_{n}$ and write $F_{n}(t)$ when no confusion will arise. As previously noted, $F_{n}\left(\mathbf{x}, \mathbf{u}, N^{(n)}(\mathbf{x}, \mathbf{u})\right)$ has an $\operatorname{Exp}(1)$ distribution, so

$$
1=\mathbb{E}\left[F_{n}\left(\mathbf{x}, \mathbf{u}, N^{(n)}(\mathbf{x}, \mathbf{u})\right)\right]
$$

and

$$
2=\mathbb{E}\left[F_{n}\left(\mathbf{x}, \mathbf{u}, N^{(n)}(\mathbf{x}, \mathbf{u})\right)^{2}\right] .
$$

In order to prove uniform convergence, we need to work on a compact set, so we fix a time $T>0$ and split the expectation and use the fact that $\mathbb{E}(\mathbf{U})=(0,0,0)$ :

$$
\begin{aligned}
0= & \mathbb{E}\left[U_{1} F_{n}\left(\mathbf{x}, \mathbf{U}, N^{(n)}(\mathbf{U})\right) \mathbb{1}_{\left\{N^{(n)}(\mathbf{U})<T\right\}}\right] \\
& +\mathbb{E}\left[U_{1} F_{n}\left(\mathbf{x}, \mathbf{U}, N^{(n)}(\mathbf{U})\right) \mathbb{1}_{\left\{N^{(n)}(\mathbf{U}) \geq T\right\}}\right]
\end{aligned}
$$


By symmetry, it is enough to consider the first coordinate. Taylor expanding $F_{n}(\mathbf{x}, \mathbf{u}, t)$ about $t=0$ yields

$$
F_{n}(\mathbf{x}, \mathbf{u}, t)=g_{n}(\mathbf{x}) v_{n}\left(r_{0}\right) t+\frac{1}{2} \ddot{F}_{n}(\mathbf{x}, \mathbf{u}, \tau(t)) t^{2}
$$

for some $\tau(t) \in[0, t]$. Here $\ddot{F}_{n}$ denotes the second derivative with respect to time $t$. Consequently

$$
\begin{aligned}
& \mathbb{E}\left[U_{1} F_{n}\left(\mathbf{x}, \mathbf{U}, N^{(n)}(\mathbf{U})\right) \mathbb{1}_{\left\{N^{(n)}(\mathbf{U})<T\right\}}\right] \\
& =g_{n}(\mathbf{x}) v_{n}(\mathbf{x}) \mathbb{E}\left[U_{1} N^{(n)}(\mathbf{U}) \mathbb{1}_{\left\{N^{(n)}(\mathbf{U})<T\right\}}\right] \\
& \quad+\frac{1}{2} \mathbb{E}\left[U_{1} \ddot{F}_{n}(\tau) N^{(n)}(\mathbf{U})^{2} \mathbb{1}_{\left\{N^{(n)}(\mathbf{U})<T\right\}}\right] .
\end{aligned}
$$

If we substitute this into (2.33) we can write

$$
\begin{aligned}
& \mathbb{E}\left[U_{1} N^{(n)}(\mathbf{U}) \mathbb{1}_{\left\{N^{(n)}(\mathbf{U})<T\right\}}\right] \\
& =\frac{-1}{g_{n}(\mathbf{x}) v_{n}(\mathbf{x})}\left[\mathbb{E}\left[U_{1} F_{n}\left(\mathbf{x}, \mathbf{U}, N^{(n)}(\mathbf{U})\right) \mathbb{1}_{\left\{N^{(n)}(\mathbf{U}) \geq T\right\}}\right]\right. \\
& \left.\quad+\frac{1}{2} \mathbb{E}\left[U_{1} \ddot{F}_{n}(\tau)\left(N^{(n)}(\mathbf{U})\right)^{2} \mathbb{1}_{\left\{N^{(n)}(\mathbf{U})<T\right\}}\right]\right]
\end{aligned}
$$

and so

$$
\begin{aligned}
n^{3 / 4} \mathbb{E}\left[U_{1} N^{(n)}(\mathbf{U})\right]= & \frac{-n^{1 / 2}}{g(\mathbf{x}) v(\mathbf{x})}\left[\mathbb{E}\left[U_{1} F_{n}\left(\mathbf{x}, \mathbf{U}^{(n)}(\mathbf{U})\right) \mathbb{1}_{\left\{N^{(n)}(\mathbf{U}) \geq T\right\}}\right]\right. \\
& \left.+\frac{1}{2} \mathbb{E}\left[U_{1} \ddot{F}_{n}(\tau)\left(N^{(n)}(\mathbf{U})\right)^{2} \mathbb{1}_{\left\{N^{(n)}(\mathbf{U})<T\right\}}\right]\right] \\
& +n^{3 / 4} \mathbb{E}\left[U_{1} N^{(n)}(\mathbf{U}) \mathbb{1}_{\left\{N^{(n)}(\mathbf{U}) \geq T\right\}}\right] .
\end{aligned}
$$

Next we compute the limit as $n \rightarrow \infty$ of each term in the expansion (2.37). The first and last term on the right-hand side can easily be seen to go to 0 as follows: By applying Corollary 2.6, it follows that

$$
\lim _{n \rightarrow \infty} \sup _{(\mathbf{x}, \mathbf{u}):\|\mathbf{x}\| \in S} n^{3 / 4} \mathbb{E}\left[N^{(n)}(\mathbf{x}, \mathbf{u}) \mathbb{1}_{\left\{N^{(n)}(\mathbf{u}) \geq T\right\}}\right]=0 .
$$

Similarly, by Cauchy-Schwarz, (2.32) and by an application of Corollary 2.5 with $k=1$ we have

$$
\begin{aligned}
n^{1 / 2} \mathbb{E} & {\left[F_{n}\left(\mathbf{x}, \mathbf{u}, N^{(n)}(\mathbf{u})\right) \mathbb{1}_{\left\{N^{(n)}(\mathbf{u}) \geq T\right\}}\right] } \\
& \leq n^{1 / 2} \sqrt{\mathbb{E}\left[F_{n}^{2}\left(\mathbf{x}, \mathbf{u}, N^{(n)}(\mathbf{u})\right)\right] \mathbb{P}\left\{N^{(n)}(\mathbf{u}) \geq T\right\}} \\
& =\sqrt{2} \sqrt{n \mathbb{P}\left\{N^{(n)}(\mathbf{u}) \geq T\right\}} \\
& \rightarrow 0
\end{aligned}
$$

uniformly for $(\mathbf{x}, \mathbf{u})$ such that $\|\mathbf{x}\| \in S$ as $n \rightarrow \infty$. 
We now deal with the middle term. Differentiating equation 2.30 twice yields

$$
\begin{aligned}
\ddot{F}_{n}(t) & =v_{n}\left(\mathbf{y}_{n}(t)\right) \nabla g_{n}\left(\mathbf{y}_{n}(t)\right) \cdot \dot{\mathbf{y}}_{n}(t)+g_{n}\left(\mathbf{y}_{n}(t)\right) \nabla v_{n}\left(\mathbf{y}_{n}(t)\right) \cdot \dot{\mathbf{y}}_{n}(t) \\
& =n^{1 / 4} \dot{\mathbf{y}}_{n}(t) \cdot\left(v_{n}\left(\mathbf{y}_{n}(t)\right) \nabla g_{n}\left(\mathbf{y}_{n}(t)\right)+g_{n}\left(\mathbf{y}_{n}(t)\right) \nabla v_{n}\left(\mathbf{y}_{n}(t)\right)\right) .
\end{aligned}
$$

To evaluate $\lim _{n \rightarrow \infty} n^{1 / 4} \dot{r}_{n}(t)$, note that by (1.2) and Lemma 2.3

$$
\begin{aligned}
\left\|n^{1 / 4} \dot{\mathbf{y}}_{n}(t)-v(\mathbf{x}) \mathbf{u}\right\| & =n^{1 / 4}\left\|\dot{\mathbf{y}}_{n}(t)-\dot{\mathbf{y}}_{n}(0)\right\| \\
& \leq n^{1 / 4} \int_{0}^{t}\left\|\ddot{\mathbf{y}}_{n}(s)\right\| d s \\
& =\frac{1}{n^{1 / 4}} \int_{0}^{t} \sqrt{n}\left\|\ddot{\mathbf{y}}_{n}(s)\right\| d s \\
& \rightarrow 0
\end{aligned}
$$

uniformly for $(\mathbf{x}, \mathbf{u}, t) \in\{\mathbf{x}:\|\mathbf{x}\| \in S\} \times \mathbf{S}^{2} \times[0, T]$ as $n \rightarrow \infty$. Differentiating (1.5) shows

$$
\nabla v(\mathbf{x})=-\frac{\nabla \mathcal{U}(\mathbf{x})}{m v(\mathbf{x})}
$$

which together with (2.40), Lemma 2.3, and the continuity of $\nabla v$ and $\nabla g$ on $\{\mathbf{x}$ : $\|\mathbf{x}\| \in S\}$ forces

$$
\begin{aligned}
\lim _{n \rightarrow \infty} \ddot{F}_{n}(t) & =\ddot{F}_{1}(0)=v(\mathbf{x}) \mathbf{u} \cdot(v(\mathbf{x}) \nabla g(\mathbf{x})+g(\mathbf{x}) \nabla v(\mathbf{x})) \\
& =\mathbf{u} \cdot\left(v(\mathbf{x})^{2} \nabla g(\mathbf{x})-g(\mathbf{x}) \frac{\nabla \mathcal{U}(\mathbf{x})}{m}\right)
\end{aligned}
$$

uniformly for $(\mathbf{x}, \mathbf{u}, t) \in\{\mathbf{x}:\|\mathbf{x}\| \in S\} \times \mathbf{S}^{2} \times[0, T]$. In conjunction with Lemma 2.7 this yields

$$
\begin{aligned}
& \sup _{(\mathbf{x}, \mathbf{u}):\|\mathbf{x}\| \in S, \mathbf{u} \in \mathbf{S}^{2}} \sqrt{n} \cdot \mathbb{E}\left[\left|\ddot{F}_{n}\left(\mathbf{x}, \mathbf{u}, N^{(n)}(\mathbf{u})\right)-\ddot{F}_{1}(\mathbf{x}, \mathbf{u}, 0)\right|\left(N^{(n)}(\mathbf{u})\right)^{2} \mathbb{1}_{\left\{N^{(n)}(\mathbf{u})<T\right\}}\right] \\
& \leq \sup _{(\mathbf{x}, \mathbf{u}):\|\mathbf{x}\| \in S, \mathbf{u} \in \mathbf{S}^{2}}\left\{\left|\ddot{F}_{n}(\mathbf{x}, \mathbf{u}, t)-\ddot{F}_{1}(\mathbf{x}, 0)\right|\right\} \sqrt{n} \\
& \quad \times \sup _{(\mathbf{x}, \mathbf{u}):\|\mathbf{x}\| \in S, \mathbf{u} \in \mathbf{S}^{2}} \mathbb{E}\left[\left(N^{(n)}(\mathbf{u})\right)^{2} \mathbb{1}_{\left\{N^{(n)}(\mathbf{u})<T\right\}}\right] \\
& \quad \rightarrow 0
\end{aligned}
$$

as $n \rightarrow \infty$. This combined with (2.28) shows

$$
\begin{array}{r}
\lim _{n \rightarrow \infty} \sup _{(\mathbf{x}, \mathbf{u}):\|\mathbf{x}\| \in S, \mathbf{u} \in \mathbf{S}^{2}} \mathbb{E}\left[\sqrt{n} \ddot{F}_{n}\left(\mathbf{x}, \mathbf{u}, N^{(n)}(\mathbf{u})\right)\left(N^{(n)}(\mathbf{u})\right)^{2} \mathbb{1}_{\left\{N^{(n)}(\mathbf{u})<T\right\}}\right. \\
\left.-\frac{2}{g^{2}(\mathbf{x}) v^{2}(\mathbf{x})} \ddot{F}_{1}(\mathbf{x}, \mathbf{u}, 0)\right]=0 .
\end{array}
$$


Combining these calculations with the expansion (2.37), we have

$$
\begin{aligned}
\lim _{n \rightarrow \infty} n^{3 / 4} \mathbb{E}\left[U_{1} N^{(n)}(\mathbf{U})\right] & =\lim _{n \rightarrow \infty} \frac{-n^{1 / 2}}{2 g(\mathbf{x}) v(\mathbf{x})} \mathbb{E}\left[U_{1} \ddot{F}_{n}(\tau)\left(N^{(n)}(\mathbf{U})\right)^{2} \mathbb{1}_{\left\{N^{(n)}(\mathbf{U})<T\right\}}\right] \\
& =\frac{-1}{g(\mathbf{x})^{3} v(\mathbf{x})^{3}} \mathbb{E}\left[U_{1} \mathbf{U} \cdot\left(v(\mathbf{x})^{2} \nabla g(\mathbf{x})-g(\mathbf{x}) \frac{\nabla \mathcal{U}(\mathbf{x})}{m}\right)\right] \\
& =\frac{-1}{3 g(\mathbf{x})^{3} v(\mathbf{x})^{3}}\left(v(\mathbf{x})^{2} g_{x_{1}}(\mathbf{x})-g(\mathbf{x}) \frac{\mathcal{U}_{x_{1}}(\mathbf{x})}{m}\right) .
\end{aligned}
$$

This completes the proof.

Let $\left(\mathbf{X}_{k}^{n}, \mathcal{T}_{k}^{n}\right)_{k \geq 0}$ be a Markov chain with transition operator

$$
Q_{n} f(\mathbf{x}, t)=\mathbb{E}\left[f\left(\mathbf{y}\left[\mathbf{x}, v(\mathbf{x}) \mathbf{U}, N^{n}(\mathbf{x}, \mathbf{U})\right], t+n^{-3 / 4} N^{n}(\mathbf{x}, \mathbf{U})\right)\right],
$$

as defined in (1.16). Note that $\left(\mathbf{X}_{k}^{n}\right)_{k \geq 0}$ is a Markov chain with transition operator (1.7) (with appropriate scaling), while the second coordinate keeps track of the time between collisions. That is, if we start the chain from $(\mathbf{x}, 0)$, then $n^{3 / 4} \mathcal{T}_{k}^{n}=T_{k}^{n}$ is the time at which the $k$ th collision occurs.

Lemma 2.10. Let

$$
\mu_{n}(\mathbf{x}, t):=n \mathbb{E}\left[\left(\mathbf{X}_{1}^{n}, \mathcal{T}_{1}^{n}\right)-(\mathbf{x}, t) \mid\left(\mathbf{X}_{0}^{n}, \mathcal{T}_{0}^{n}\right)=(\mathbf{x}, t)\right]
$$

be the scaled drift of $\left(\mathbf{X}_{k}^{n}, \mathcal{T}_{k}^{n}\right)_{k \geq 0}$. Then

$$
\lim _{n \rightarrow \infty} \sup _{(\mathbf{x}, t):\|\mathbf{x}\| \in S, t \geq 0}\left|\mu_{n}(\mathbf{x}, t)-\left(\frac{-1}{3 g(\mathbf{x})^{2}}\left[\frac{2 \nabla \mathcal{U}(\mathbf{x})}{m v(\mathbf{x})^{2}}+\frac{\nabla g(\mathbf{x})}{g(\mathbf{x})}\right], \frac{1}{g(\mathbf{x}) v(\mathbf{x})}\right)\right|=0
$$

uniformly on $\{\mathbf{x}:\|\mathbf{x}\| \in S\} \times[0, \infty)$.

Proof. The result for the time coordinate is simply a restatement of (2.27). By symmetry, it suffices to prove convergence for the first spatial coordinate only. By definition of $X_{1}^{n}$, this is

$$
n \mathbb{E}\left[X_{1}^{n}-x_{1} \mid\left(\mathbf{X}_{0}^{n}, \mathcal{T}_{0}^{n}\right)=(\mathbf{x}, t)\right]=n \mathbb{E}\left[y_{n, 1}\left(N^{(n)}(\mathbf{U})\right)-x_{1}\right] .
$$

Let $\Lambda_{\delta}(S):=\Lambda_{\delta}^{1}(S)$ as defined in (2.6). Since $\Lambda_{\delta}^{n}(S)$ is increasing in $n$, we have by construction that $\left\|\mathbf{y}_{n}(t)\right\|=r_{n}(t) \in S^{\prime}$ for all $n \in \mathbb{N}$ and $t \in\left[0, \Lambda_{\delta}(S)\right]$. So, in particular, $\mathbf{y}_{n}(t)$ is bounded away from $\partial \mathcal{D}$. To compute $\mu_{n, 1}$ we first split the expectation on the events $\left\{N^{(n)}(\mathbf{U}) \leq \Lambda_{\delta}(S)\right\}$ and $\left\{N^{(n)}(\mathbf{U})>\Lambda_{\delta}(S)\right\}$. This allows us to write

$$
\begin{aligned}
n \mathbb{E}\left[y_{n, 1}\left(N^{(n)}(\mathbf{U})\right)-x_{1}\right] & =n \mathbb{E}\left[\left(y_{n, 1}\left(N^{(n)}(\mathbf{U})\right)-x_{1}\right) \mathbb{1}_{\left\{N^{(n)} \leq \Lambda_{\delta}(S)\right\}}\right] \\
& +n \mathbb{E}\left[\left(y_{n, 1}\left(N^{(n)}(\mathbf{U})\right)-x_{1}\right) \mathbb{1}_{\left\{N^{(n)}>\Lambda_{\delta}(S)\right\}}\right] .
\end{aligned}
$$

By Lemma 2.8, the second term of (2.44) converges to 0 uniformly on $\{\mathbf{x}:\|\mathbf{x}\| \in S\}$.

To compute the first term of (2.44), we utilize a second order Taylor expansion of $y_{n, 1}(t)$ evaluated at $t=N^{(n)}(\mathbf{U})$ which yields

$$
y_{n, 1}\left(\mathbf{x}, N^{(n)}(\mathbf{U})\right)-x_{1}=v_{n}(\mathbf{x}) U_{1} N^{(n)}(\mathbf{U})+\frac{1}{2} \ddot{y}_{n, 1}(\tau)\left[N^{(n)}(\mathbf{U})\right]^{2}
$$


for some $0 \leq \tau:=\tau\left(\mathbf{x}, N^{n}(\mathbf{U})\right) \leq N^{n}(\mathbf{U})$. Hence

$$
\begin{aligned}
n \mathbb{E}\left[\left(y_{n, 1}\left(N^{(n)}(\mathbf{U})\right)-x_{1}\right) \mathbb{1}_{\left\{N^{(n)} \leq \Lambda_{\delta}(S)\right\}}\right] \\
=\mathbb{E}\left[n v_{n}(\mathbf{x}) U_{1} N^{(n)}(\mathbf{U}) \mathbb{1}_{\left\{N^{(n)} \leq \Lambda_{\delta}(S)\right\}}\right] \\
+\frac{n}{2} \mathbb{E}\left[\ddot{y}_{n, 1}(\tau) N^{(n)}(\mathbf{U})^{2} \mathbb{1}_{\left\{N^{(n)} \leq \Lambda_{\delta}(S)\right\}}\right] .
\end{aligned}
$$

Note that

$$
\begin{aligned}
& \left|\mathbb{E}\left[n v_{n}(\mathbf{x}) U_{1} \cdot N^{(n)}(\mathbf{U}) \mathbb{1}_{\left\{N^{(n)}>\Lambda_{\delta}(S)\right\}}\right]\right| \\
& \quad \leq \sup _{S}|v(\mathbf{x})| n^{3 / 4} \mathbb{E}\left[N^{(n)}(\mathbf{U}) \mathbb{1}_{\left\{N^{(n)}>\Lambda_{\delta}(S)\right\}}\right] \rightarrow 0
\end{aligned}
$$

uniformly for $\mathbf{x}$ such that $\|\mathbf{x}\| \in S$ as $n \rightarrow \infty$ by Corollary 2.6. Thus the limit of the first term of (2.45) can be computed by a direct application of Lemma 2.9.

$$
\begin{aligned}
& \lim _{n \rightarrow \infty} \mathbb{E}\left[n v_{n}(\mathbf{x}) U_{1} \cdot N^{(n)}(\mathbf{U}) \mathbb{1}_{\left\{N^{(n)} \leq \Lambda_{\delta}(S)\right\}}\right] \\
& =\lim _{n \rightarrow \infty} v(\mathbf{x}) \mathbb{E}\left[n^{3 / 4} U_{1} \cdot N^{(n)}(\mathbf{U})\right]=\frac{1}{3 g(\mathbf{x})^{2}}\left(\frac{\mathcal{U}_{x_{1}}(\mathbf{x})}{m v(\mathbf{x})^{2}}-\frac{g_{x_{1}}(\mathbf{x})}{g(\mathbf{x})}\right)
\end{aligned}
$$

uniformly on $\{\mathbf{x}:\|\mathbf{x}\| \in S\}$. We now compute the limit of the second term on the right-hand side of (2.45).

Since $\tau\left(N^{(n)}(\theta)\right) \leq N^{(n)}(\theta)$, by Lemma 2.3 and Lemma 2.4

$$
\begin{array}{r}
\sup _{(\mathbf{x}, \mathbf{u}):\|\mathbf{x}\| \in S, \mathbf{u} \in \mathbf{S}^{2}}\left|\mathbb{E}\left[\left(\sqrt{n} \ddot{y}_{n, 1}\left(\tau\left(N^{(n)}(\mathbf{u})\right)\right)+\frac{\mathcal{U}_{x_{1}}(\mathbf{x})}{m}\right) \sqrt{n}\left(N^{(n)}(\mathbf{u})\right)^{2} \mathbb{1}_{\left\{N^{(n)} \leq \Lambda_{\delta}(S)\right\}}\right]\right| \\
\leq \sup _{(\mathbf{x}, \mathbf{u}):\|\mathbf{x}\| \in S, \mathbf{u} \in \mathbf{S}^{2}}\left\{\sup _{t \in\left[0, \Lambda_{\delta}(S)\right]}\left|\sqrt{n} \ddot{y}_{n, 1}(\mathbf{x}, \mathbf{u}, t)+\frac{\mathcal{U}_{x_{1}}(\mathbf{x})}{m}\right|\right. \\
\left.\cdot \mathbb{E}\left[\sqrt{n} N^{(n)}(\mathbf{u})^{2} \mathbb{1}_{\left\{N^{(n)} \leq \Lambda_{\delta}(S)\right\}}\right]\right\} \rightarrow 0
\end{array}
$$

as $n \rightarrow \infty$. This together with Corollary 2.6 and Lemma 2.7 yields

$$
\begin{array}{rl}
\lim _{n \rightarrow \infty} \sup _{(\mathbf{x}, \mathbf{u}):\|\mathbf{x}\| \in S, \mathbf{u} \in \mathbf{S}^{2}} & \mathbb{E}\left[n \ddot{y}_{n, 1}\left(\tau\left(N^{(n)}(\mathbf{u})\right)\right) N^{(n)}(\mathbf{u})^{2} \mathbb{1}_{\left\{N^{(n)} \leq \Lambda_{\delta}(S)\right\}}\right] \\
& +\frac{2 \mathcal{U}_{x_{1}}(\mathbf{x})}{m g(\mathbf{x})^{2} v(\mathbf{x})^{2}} \mid=0 .
\end{array}
$$

Since this convergence is uniform in $\mathbf{u}$, we can evaluate the limit of the second order term of equation (2.45) by

$$
\lim _{n \rightarrow \infty} \mathbb{E}\left[\frac{n}{2} \ddot{y}_{n, 1}\left(\tau\left(N^{(n)}(\mathbf{U})\right)\right) N^{(n)}(\mathbf{U})^{2} \mathbb{1}_{\left\{N^{(n)}(\mathbf{U}) \leq \Lambda_{\delta}(S)\right\}}\right]=-\frac{\mathcal{U}_{x_{1}}(\mathbf{x})}{m g(\mathbf{x})^{2} v(\mathbf{x})^{2}}
$$

uniformly on $\{\mathbf{x}:\|\mathbf{x}\| \in S\}$. So by adding the right-hand sides of (2.46) and (2.47) we have

$$
\lim _{n \rightarrow \infty} n \mathbb{E}\left[X_{1}^{n}-x_{1} \mid\left(\mathbf{X}_{0}^{n}, \mathcal{T}_{0}^{n}\right)=(\mathbf{x}, t)\right]=-\frac{1}{3 g(\mathbf{x})^{2}}\left(\frac{2 \mathcal{U}_{x_{1}}(\mathbf{x})}{m v(\mathbf{x})^{2}}+\frac{g_{x_{1}}(\mathbf{x})}{g(\mathbf{x})}\right)
$$

uniformly on $\{\mathbf{x}:\|\mathbf{x}\| \in S\} \times[0, \infty)$. 
Lemma 2.11. Let $\left(\left(\mathbf{X}_{k}^{n}, \mathcal{T}_{k}^{n}\right)\right)_{k \geq 0}$ be a Markov chain with transition operator as in (1.16) and let

$$
\begin{aligned}
\sigma_{n, i j}^{2}(\mathbf{x}, t) & :=n \mathbb{E}\left[\left(X_{1, i}^{n}-x_{i}\right)\left(X_{1, j}^{n}-x_{j}\right) \mid\left(\mathbf{X}_{0}^{n}, \mathcal{T}_{0}^{n}\right)=(\mathbf{x}, t)\right] \\
\sigma_{n, i t}^{2}(\mathbf{x}, t) & :=n \mathbb{E}\left[\left(X_{1, i}^{n}-x_{i}\right)\left(\mathcal{T}_{1}^{n}-t\right) \mid\left(\mathbf{X}_{0}^{n}, \mathcal{T}_{0}^{n}\right)=(\mathbf{x}, t)\right] \\
\sigma_{n, t}^{2}(\mathbf{x}, t) & :=n \mathbb{E}\left[\left(\mathcal{T}_{1}^{n}-t\right)^{2} \mid\left(\mathbf{X}_{0}^{n}, \mathcal{T}_{0}^{n}\right)=(\mathbf{x}, t)\right] .
\end{aligned}
$$

Then

(1) $\lim _{n \rightarrow \infty} \sup _{(\mathbf{x}, t):\|\mathbf{x}\| \in S, t \geq 0}\left|\sigma_{n, i j}^{2}(\mathbf{x}, t)-\frac{2}{3 g(\mathbf{x})^{2}} \delta_{i j}\right|=0$, where $\delta_{i j}=\mathbb{1}\{i=j\}$.

(2) $\lim _{n \rightarrow \infty} \sup _{(\mathbf{x}, t):\|\mathbf{x}\| \in S, t \geq 0} \sigma_{n, i t}^{2}(\mathbf{x}, t)=0$.

(3) $\lim _{n \rightarrow \infty} \sup _{(\mathbf{x}, t):\|\mathbf{x}\| \in S, t \geq 0} \sigma_{n, t}^{2}(\mathbf{x}, t)=0$.

Proof. Note that (3) is an immediate consequence of Lemma 2.7 while (2) follows from (1), (3) and an application of Cauchy-Schwarz. It remains to establish (1). As in the proof of Lemma 2.10 we have

$$
\begin{aligned}
\sigma_{n, i j}^{2}(\mathbf{x}, t)=n \mathbb{E} & {\left[\left(y_{n, i}\left(N^{(n)}(\mathbf{U})\right)-x_{i}\right)\left(y_{n, j}\left(N^{(n)}(\mathbf{U})\right)-x_{j}\right) \mathbb{1}_{\left\{N^{(n)} \leq \Lambda_{\delta}(S)\right\}}\right] } \\
& +n \mathbb{E}\left[\left(y_{n, i}\left(N^{(n)}(\mathbf{U})\right)-x_{i}\right)\left(y_{n, j}\left(N^{(n)}(\mathbf{U})\right)-x_{j}\right) \mathbb{1}_{\left\{N^{(n)}>\Lambda_{\delta}(S)\right\}}\right] .
\end{aligned}
$$

The second term on the right-hand side goes to 0 by Lemma 2.8. To evaluate the limit of the first term on the right-hand side of (2.48), it is enough to use a first order Taylor expansion of $\mathbf{y}_{n}(t)$ :

$$
y_{n, i}\left(\mathbf{x}, N^{(n)}(\mathbf{U})\right)-x_{i}=\dot{y}_{n, i}(\tau) N^{(n)}(\mathbf{U})
$$

for some $0 \leq \tau:=\tau\left(\mathbf{x}, N^{n}(\mathbf{U})\right) \leq N^{n}(\mathbf{U})$. Hence

$$
\begin{aligned}
n \mathbb{E}\left[\left(y_{n, i}\left(N^{(n)}(\mathbf{U})\right)-x_{i}\right)\right. & \left.\left(y_{n, j}\left(N^{(n)}(\mathbf{U})\right)-x_{j}\right) \mathbb{1}_{\left\{N^{(n)} \leq \Lambda_{\delta}(S)\right\}}\right] \\
& =n \mathbb{E}\left[\dot{y}_{n, i}(\tau) \dot{y}_{n, j}(\tau) N^{(n)}(\mathbf{U})^{2} \mathbb{1}_{\left\{N^{(n)} \leq \Lambda_{\delta}(S)\right\}}\right]
\end{aligned}
$$

Note that for $t \leq \Lambda_{\delta}(S)$ and $\mathbf{x}$ such that $\|\mathbf{x}\| \in S,\left\|\mathbf{y}_{n}(\mathbf{x}, \mathbf{u}, t)\right\| \in S^{\prime}$. By the mean value theorem and the fact that $\nabla \mathcal{U}$ is bounded on $\left\{\mathbf{z}:\|\mathbf{z}\| \in S^{\prime}\right\}$, there exists $C>0$ such that

$$
\begin{gathered}
\sup _{(\mathbf{x}, \mathbf{u}):\|\mathbf{x}\| \in S, \mathbf{u} \in \mathbf{S}^{2}} \sup _{t \in\left[0, \Lambda_{\delta}(S)\right]}\left|n^{1 / 4} \dot{y}_{n, i}(\mathbf{x}, \mathbf{u}, \tau)-v(\mathbf{x}) u_{i}\right| \\
\leq \frac{2}{m n^{1 / 4}} \sup _{\mathbf{z}:\|\mathbf{z}\| \in S^{\prime}} \mathcal{U}_{x_{i}}(\mathbf{z}) \Lambda_{\delta}(S) \leq \frac{C}{n^{1 / 4}}
\end{gathered}
$$


Since $\tau\left(N^{(n)}(\theta)\right) \leq N^{(n)}(\theta)$, we can apply Lemma 2.4 and Lemma 2.7 to see that

$$
\begin{aligned}
\lim _{n \rightarrow \infty} & n \mathbb{E}\left[\dot{y}_{n, i}(\tau) \dot{y}_{n, j}(\tau) N^{(n)}(\mathbf{U})^{2} \mathbb{1}_{\left\{N^{(n)} \leq \Lambda_{\delta}(S)\right\}}\right] \\
& =\lim _{n \rightarrow \infty} v(\mathbf{x})^{2} \mathbb{E}\left[U_{i} U_{j}\left(n^{1 / 2} N^{(n)}(\mathbf{U})^{2}\right)\right] \\
& =\frac{2}{g(\mathbf{x})^{2}} \mathbb{E}\left(U_{i} U_{j}\right) \\
& =\frac{2}{3 g(\mathbf{x})^{2}} \delta_{i j}
\end{aligned}
$$

uniformly on $\{\mathbf{x}:\|\mathbf{x}\| \in S\}$, which completes the proof.

\section{Convergence of the Free PATH PROCESS}

In this section we will prove the convergence of the free path process $\left(\mathbf{X}_{k}^{n}, \mathcal{T}_{k}^{n}\right)_{k \in \mathbb{N}_{0}}$ with transition operator $Q_{n}$ defined in (1.16). Recall that $\mathcal{D}_{3}=\left\{\mathbf{x} \in \mathbb{R}^{3}:\|\mathbf{x}\| \in \mathcal{D}\right\}$. The following is the main theorem of this section.

Theorem 3.1. Let $\left(\mathcal{X}_{t}\right)_{t \geq 0}$ be a diffusion on $\mathcal{D}_{3}$ whose generator $G$ acts on functions $f \in C^{2}\left(\mathcal{D}_{3}\right)$ with compact support in $\mathcal{D}_{3}^{\circ}$ by

$$
G f(\mathbf{x})=\frac{1}{3 g^{2}(\mathbf{x})} \Delta f(\mathbf{x})-\frac{1}{3 g^{2}(\mathbf{x})}\left(\frac{\nabla g(\mathbf{x})}{g(\mathbf{x})}+\frac{2 \nabla \mathcal{U}(\mathbf{x})}{m v(\mathbf{x})^{2}}\right) \cdot \nabla f(\mathbf{x})
$$

and killed if/when the diffusion $\mathcal{R}:=\|\mathcal{X}\|$ hits the boundary of $\mathcal{D}$. Consider any $l, u \in \mathcal{D}^{\circ}$ with $l<u$ and start the process $\left(\mathbf{X}_{k}^{n}, \mathcal{T}_{k}^{n}\right)_{k \in \mathbb{N}_{0}}$ at $(\mathbf{x}, 0)$, where $l<\|\mathbf{x}\|<u$. Define the stopping times

$$
\tau_{l, u}^{n}:=\inf \left\{k \in \mathbb{N}_{0}:\left\|\mathbf{X}_{k}^{n}\right\| \notin[l, u]\right\}
$$

and

$$
\tau_{l, u}:=\inf \left\{t \geq 0:\left\|\mathcal{X}_{t}\right\| \notin[l, u]\right\} .
$$

Then, as $n \rightarrow \infty$, the family of continuous time processes $\left(\mathbf{X}_{\lfloor n t\rfloor \wedge \tau_{l, u}^{n}}^{n}, \mathcal{T}_{\lfloor n t\rfloor \wedge \tau_{l, u}^{n}}^{n}\right)_{t \geq 0}$ converges in distribution on the Skorokhod space to the diffusion

$$
\left(\mathcal{X}_{t \wedge \tau_{l, u}}, \int_{0}^{t \wedge \tau_{l, u}} \frac{d s}{g\left(\mathcal{X}_{s}\right) v\left(\mathcal{X}_{s}\right)}\right)_{t \geq 0}
$$

with initial conditions $(\mathbf{x}, 0)$.

As an immediate corollary we get the following result for the diffusion $\mathcal{R}$.

Corollary 3.2. Suppose $\mathcal{X}$ is the diffusion defined in Theorem 3.1 , Then $\mathcal{R}:=\|\mathcal{X}\|$ is a diffusion on $\mathcal{D}^{\circ}$ with a generator $G_{r}$ that acts on functions $f \in C^{2}(\mathcal{D})$ with compact support in $\mathcal{D}^{\circ}$ by

$$
G_{r} f(r)=\frac{1}{3 g^{2}(r)} \partial_{r r}^{2} f(r)-\frac{1}{3 g^{2}(r)}\left(\frac{\partial_{r} g(r)}{g(r)}-\frac{1}{r}+\frac{2 \partial_{r} U(r)}{m v^{2}(r)}\right) \partial_{r} f(r) .
$$

Proof. This follows by applying Ito's Lemma to the diffusion $\mathcal{X}$ from (3.1) together with the following two observations:

(a) If $g(\mathbf{x})$ only depends on $\|x\|=r$, then

$$
\nabla g(\mathbf{x})=\partial_{r} g(r) \frac{\mathbf{x}}{r} .
$$


(b) If $\mathcal{X}=:\left(X_{1}, X_{2}, X_{3}\right)$ and $\left(B_{1}, B_{2}, B_{3}\right)$ is a standard three-dimensional Brownian motion, then

$$
\sum_{i=1}^{3} X_{i} d B_{i} / \mathcal{R}
$$

is a standard one-dimensional Brownian motion. This follows from the fact that

$$
d\left[\sum_{i=1}^{3} X_{i} d B_{i} / \mathcal{R}\right]_{t}=\sum_{i=1}^{3} \frac{X_{i}^{2}}{\mathcal{R}^{2}} d t=d t
$$

We prove Theorem 3.1 at the end of this section. The proof utilizes Theorem IX.4.21 from JS03 which gives sufficient conditions to prove that a continuous time step process converges to a diffusion. We add this result below in order to make it easier for the reader to follow our proof.

Theorem 3.3. Suppose that for each $n \in \mathbb{N}, X^{n}$ is a pure jump Markov process. That is, its generator has the form

$$
A^{n} f(x)=\int[f(x+y)-f(x)] K^{n}(x, d y),
$$

where $K^{n}$ is a finite transition kernel on $\mathbb{R}^{d}$. Then define $b^{n}$ and $c^{n}$ by

$$
b^{n}(x)=\int y K^{n}(x, d y), c^{n, i j}(x)=\int y^{i} y^{j} K^{n}(x, d y) .
$$

Let $b, c$ be continuous functions on $\mathbb{R}^{d}$ and suppose $X$ is a diffusion whose generator is given by

$$
G f(x)=\sum_{i \leq d} b_{i}(x) D_{i} f(x)+\frac{1}{2} \sum_{1 \leq i, j \leq d} c^{i j}(x) D_{i j} f(x)
$$

and defines a martingale problem with a unique solution (see Assumption IX.4.3 from [JS03]). Assume that

(i) $b^{n} \rightarrow b, c^{n} \rightarrow c$ locally uniformly;

(ii) $\sup _{x:|x| \leq a} \int K^{n}(x, d y)|y|^{2} \mathbb{1}_{\{|y|>\varepsilon\}} \rightarrow 0$ as $n \uparrow \infty$ for all $\varepsilon>0$;

(iii) $\nu_{n} \rightarrow \nu$ weakly, where $\nu_{n}$ and $\nu$ are the initial distributions of $X_{0}^{n}$ and $X_{0}$ respectively.

Then the laws $\mathcal{L}\left(X^{n}\right)$ converge weakly to $P=\int P_{x} \nu(d x)$, the law of the diffusion process $X$ started with the initial distribution $\nu$.

Because we need to stop the diffusion before it enters a domain where the particle's velocity is not bounded away from 0 , the easiest way to apply Theorem 3.3 to our situation is to introduce a cutoff version of the chains $\left(\left(\mathbf{X}_{k}^{n}, \mathcal{T}_{k}^{n}\right)\right)_{k \geq 0}$, that behaves like this chain on $\{(\mathbf{x}, t): l \leq\|\mathbf{x}\| \leq u\}$ and obviously satisfies the conditions of Theorem 3.3 outside this domain. To this end, fix $l^{*}, u^{*} \in \mathcal{D}^{\circ}$ such that $l^{*}<l<u<u^{*}$, and let $\phi: \mathbb{R} \rightarrow[0,1]$ be a smooth function such that $\phi$ is identically 0 in a neighborhood of 0 and in a neighborhood of $\infty$, and $\phi(r)=1$ if $r \in\left[l^{*}, u^{*}\right]$, and let $\zeta(\mathbf{x})=(\|\mathbf{x}\| \vee l) \wedge u$. Moreover, define

$$
b^{l u}(\mathbf{x}, t)=b^{l u}(\mathbf{x})=\left(\frac{-1}{3 g(\zeta(\mathbf{x}))^{2}}\left[\frac{2 \nabla(\phi \mathcal{U})(\mathbf{x})}{m v(\zeta(\mathbf{x}))^{2}}+\frac{\nabla(\phi g)(\mathbf{x})}{g(\zeta(\mathbf{x}))}\right], \frac{1}{g(\zeta(\mathbf{x})) v(\zeta(\mathbf{x}))}\right)
$$


and

$$
c_{i j}^{l u}(\mathbf{x}, t)=c_{i j}^{l u}(\mathbf{x})=\frac{2}{3 g(\zeta(\mathbf{x}))^{2}} \delta_{i j} \mathbb{1}(i \leq 3) .
$$

Let $\mathbf{A}$ be uniformly distributed on $\{-1,1\}^{3}$, and define the transition operator $R_{n}^{l u}$ by

$$
R_{n}^{l u} f(\mathbf{x}, t)=\mathbb{E}\left[f\left((\mathbf{x}, t)+n^{-1 / 2} \sqrt{c_{11}^{l u}(\mathbf{x})}(\mathbf{A}, 0)+n^{-1} b^{l u}(\mathbf{x})\right)\right] .
$$

The next two results follow easily from these definitions.

Proposition 3.4. Let $\mathcal{G}_{l u}$ act on $f \in C^{2}\left(\mathbb{R}^{4}\right)$ with compact support by

$$
\mathcal{G}_{l u} f(\mathbf{x}, t)=\sum_{i=1}^{3} \frac{c_{i i}^{l u}(\mathbf{x})}{2} D_{i i} f(\mathbf{x}, t)+\sum_{i=1}^{4} b_{i}^{l u}(\mathbf{x}) D_{i} f(\mathbf{x}, t) .
$$

The martingale problem for $\mathcal{G}_{l u}$ is well posed.

Proof. The generator $\mathcal{G}_{l u}$ is slightly degenerate because $c_{44}^{l u} \equiv 0$, but this is not a serious complication, as is easily seen from the SDE perspective. In particular, note that if $\left(Z_{t}^{1}, Z_{t}^{2}, Z_{t}^{3}, Z_{t}^{4}\right)_{t \geq 0}$ solves the corresponding SDE

$$
d Z^{i}=\sqrt{c_{i i}^{l u}\left(Z^{1}, Z^{2}, Z^{3}\right)} d B^{i}+b^{l u}\left(Z^{1}, Z^{2}, Z^{3}\right)_{i} d t
$$

for $1 \leq i \leq 3$ and

$$
d Z^{4}=b^{l u}\left(Z^{1}, Z^{2}, Z^{3}\right){ }_{4} d t
$$

then $Z^{4}$ is a measurable, deterministic function of $\left(Z^{1}, Z^{2}, Z^{3}\right)$ and $Z^{4}$ does not appear in the SDE for $\left(Z^{1}, Z^{2}, Z^{3}\right)$. Thus we need only establish existence and uniqueness in law for the $\operatorname{SDE}(3.6)$ for $\left(Z^{1}, Z^{2}, Z^{3}\right)$. However, this is standard since the drift coefficients are bounded and continuous and the diffusion matrix is continuous and positive definite; see e.g. [EK86, Theorem 8.1.7].

Proposition 3.5. Let $\left(\mathbf{Z}_{k}^{n}\right)_{k \geq 0}$ be a Markov chain on $\mathbb{R}^{4}$ with transition operator $R_{n}^{l u}$. Then, uniformly on $\mathbb{R}^{4}$, we have

(1) $\lim _{n \rightarrow \infty} n \mathbb{E}\left(\mathbf{Z}_{1}^{n}-\mathbf{z} \mid \mathbf{Z}_{0}^{n}=\mathbf{z}\right)=b^{l u}(\mathbf{z})$,

(2) $\lim _{n \rightarrow \infty} n \mathbb{E}\left(\left(Z_{1, i}^{n}-z_{i}\right)\left(Z_{1, j}^{n}-z_{j}\right) \mid \mathbf{Z}_{0}^{n}=\mathbf{z}\right)=c_{i j}^{l u}(\mathbf{z})$,

(3) $\lim _{n \rightarrow \infty} \max _{k \geq 0}\left\|\mathbf{Z}_{k+1}^{n}-\mathbf{Z}_{k}^{n}\right\|=0$ almost surely.

To handle the cutoff, we will need the following elementary result about convergence of hitting times with respect to the Skorokhod topology.

Lemma 3.6. For $\gamma \in D[0, \infty)$ define $\tau_{x}(\gamma)=\inf \{t: \gamma(t) \geq x\}$ to be the first passage time of $\gamma$ across $x$. Consider an $f \in C[0, \infty)$ and an $a>0$ such that

$$
0<\tau_{a}(f)<\infty \quad \text { and } \inf \left\{t>\tau_{a}(f): f(t)>a\right\}=\tau_{a}(f) .
$$

If $\left(f_{n}\right)_{n \geq 1}$ is a sequence in $D[0, \infty)$ such that $f_{n} \rightarrow f$ in $D[0, \infty)$, then $\tau_{a}\left(f_{n}\right) \rightarrow$ $\tau_{a}(f)$.

Proof of Theorem 3.1. Let us introduce the cutoff transition operator $Q_{n}^{l u}$ defined by

$$
Q_{n}^{l u} f(\mathbf{x}, t)= \begin{cases}Q_{n}(\mathbf{x}, t) & \text { if }\|\mathbf{x}\| \in[l, u] \\ R_{n}^{l u}(\mathbf{x}, t) & \text { if }\|\mathbf{x}\| \notin[l, u]\end{cases}
$$


Let $\left(\mathbf{X}_{k}^{l u, n}, \mathcal{T}_{k}^{l u, n}\right)_{k \geq 0}$ be a Markov chain with transition operator $Q_{n}^{l u} f(\mathbf{x}, t)$ started from $(\mathbf{x}, 0)$ with $\|\mathbf{x}\| \in(l, u)$ and let $\eta_{n}=\inf \left\{k:\left\|\mathbf{X}_{k}^{l u, n}\right\| \notin[l, u]\right\}$. By the definition of the cutoff transition operator, we have the identity in distribution

$$
\left(\mathbf{X}_{k \wedge \eta_{n}}^{l u, n}, \mathcal{T}_{k \wedge \eta_{n}}^{l u, n}\right)_{k \geq 0}={ }_{d}\left(\mathbf{X}_{k \wedge \tau_{l, u}^{n}}^{n}, \mathcal{T}_{k \wedge \tau_{l, u}^{n}}^{n}\right)_{k \geq 0}
$$

so it suffices to prove the result for the cutoff chain $\left(\mathbf{X}_{k}^{l u, n}, \mathcal{T}_{k}^{l u, n}\right)_{k \geq 0}$. In order to apply Theorem 3.3 directly, we Poissonize the chain. That is, let $\left(\Gamma_{t}\right)_{t \geq 0}$ be a rate one Poisson process and consider

$$
\left(\mathbf{X}^{l u, n}(t), \mathcal{T}^{l u, n}(t)\right)_{t \geq 0}:=\left(\mathbf{X}_{\Gamma_{t}}^{l u, n}, \mathcal{T}_{\Gamma_{t}}^{l u, n}\right)_{t \geq 0}
$$

Since $\Gamma_{n t} / n \rightarrow t$ uniformly on compact sets almost surely, it is enough to prove the result for $\left(\mathbf{X}^{l u, n}(t), \mathcal{T}^{l u, n}(t)\right)_{t \geq 0}$, where the relevant stopping time is now

$$
\eta_{n}^{c}=\inf \left\{t:\left\|\mathbf{X}^{l u, n}(t)\right\| \notin[l, u]\right\} .
$$

Note that $\left(\mathbf{X}^{l u, n}(n t), \mathcal{T}^{l u, n}(n t)\right)_{t \geq 0}$ is a pure jump Markov process with generator

$$
\mathcal{G}_{n}^{l u} f(\mathbf{x}, t)=n\left(Q_{n}^{l u}-I\right) f(\mathbf{x}, t) ;
$$

see e.g. Kal02, Proposition 19.2]. For this process, condition (i) of Theorem 3.3 is satisfied with $b=b^{l u}$ and $c=c^{l u}$ by a direct combination of Proposition 3.5. Lemma 2.10, and Lemma 2.11. That condition (ii) of Theorem 3.3 is satisfied is an immediate consequence of Proposition 3.5. Lemma 2.11 (for the time component), and Lemma 2.8, where to apply the last lemma we note that, because velocity is bounded away from 0 and $\infty$ on $[l, u]$, the condition that $N^{(n)}>\epsilon$ in Lemma 2.8 is comparable to the condition that the size of the jump be at least $\epsilon$ in condition (ii) of Theorem 3.3. Consequently we have

$$
\left(\mathbf{X}^{l u, n}(n t), \mathcal{T}^{l u, n}(n t)\right)_{t \geq 0} \Rightarrow\left(\mathcal{X}_{t}^{l u}, \mathcal{T}_{t}^{l u}\right)_{t \geq 0}
$$

where $\left(\mathcal{X}_{t}^{l u}, \mathcal{T}_{t}^{l u}\right)_{t>0}$ is a diffusion with generator (3.5) started from $(\mathbf{x}, 0)$. Using the Skorokhod representation, we may assume this convergence happens almost surely. It follows from Girsanov's theorem that $\left\|\mathcal{X}^{l u}\right\|$ satisfies the hypotheses of Lemma 3.6 and, as a result, this convergence happens jointly with the convergence of $\eta_{n}^{c} / n$ to $\eta^{l u}=\inf \left\{t:\left\|\mathcal{X}^{l u}\right\| \notin[l, u]\right\}$. Therefore

$$
\left(\mathbf{X}^{l u, n}\left((n t) \wedge \eta_{n}^{c}\right), \mathcal{T}^{l u, n}\left((n t) \wedge \eta_{n}^{c}\right)\right)_{t \geq 0} \Rightarrow\left(\mathcal{X}_{t \wedge \eta^{l u}}^{l u}, \mathcal{T}_{t \wedge \eta^{l u}}^{l u}\right)_{t \geq 0}
$$

as $n \rightarrow \infty$. From the SDE perspective (see Proposition 3.4), it is clear that the generator for $\left(\left(\mathcal{X}_{t}^{l u}, \mathcal{T}_{t}^{l u}\right)\right)_{t \geq 0}$ agrees with the generator for

$$
\left(\mathcal{X}_{t}, \int_{0}^{t} \frac{d s}{g\left(\mathcal{X}_{s}\right) v\left(\mathcal{X}_{s}\right)}\right)_{t \geq 0}
$$

on smooth functions $f(\mathbf{x}, t)$ with compact support in $\left\{\mathbf{x}:\|\mathbf{x}\| \in\left[l^{*}, u^{*}\right]\right\} \times \mathbb{R}$, and thus we have

$$
\left(\mathcal{X}_{t \wedge \eta^{l u}}^{l u}, \mathcal{T}_{t \wedge \eta^{l u}}^{l u}\right)_{t \geq 0}={ }_{d}\left(\mathcal{X}_{t \wedge \tau_{l, u}}, \int_{0}^{t \wedge \tau_{l, u}} \frac{d s}{g\left(\mathcal{X}_{s}\right) v\left(\mathcal{X}_{s}\right)}\right)_{t \geq 0}
$$


As a result of de-Poissonizing the process and (3.8), we have

$$
\left(\mathbf{X}_{\lfloor n t\rfloor \wedge \tau_{l, u}^{n}}^{n}, \mathcal{T}_{\lfloor n t\rfloor \wedge \tau_{l, u}^{n}}^{n}\right)_{t \geq 0} \Rightarrow\left(\mathcal{X}_{t \wedge \tau_{l, u}}, \int_{0}^{t \wedge \tau_{l, u}} \frac{d s}{g\left(\mathcal{X}_{s}\right) v\left(\mathcal{X}_{s}\right)}\right)_{t \geq 0} .
$$

4. The PROCESS ON ITS NATURAL TIME SCALE

In this section, we study the convergence of the full trajectory of the particle. To reconstruct the full path from the free path process, we also need to keep track of the direction of reflection. To this end, we need to keep track of the reflection times and the direction of reflection. That is, we will look at the Markov process $\left(\mathbf{X}_{k}^{n}, \mathcal{T}_{k}^{n}, \mathbf{U}_{k}^{n}\right)_{k \in \mathbb{N}_{0}}$ started from $(\mathbf{x}, 0,0)$ with $\|\mathbf{x}\| \in \mathcal{D}$ and transition operator

$$
\hat{Q}_{n} f(\mathbf{x}, t, \mu)=\mathbb{E}\left[f\left(\mathbf{y}\left[\mathbf{x}, v(\mathbf{x}) \mathbf{U}, N^{n}(\mathbf{x}, \mathbf{U})\right], t+n^{-3 / 4} N^{n}(\mathbf{x}, \mathbf{U}), \mathbf{U}\right)\right] .
$$

By (1.4) the full trajectory $\left(\mathbf{X}^{n}(t)\right)_{t \geq 0}$ of the particle with energy $E_{n}$ moving in potential $\mathcal{U}_{n}$ and scattering density $g_{n}$ is then constructed by

$$
\mathbf{X}^{n}(t):=\mathbf{y}_{n}\left(\mathbf{X}_{k}^{n}, v\left(\mathbf{X}_{k}^{n}\right) \mathbf{U}_{k}^{n}, t-n^{3 / 4} \mathcal{T}_{k}^{n}\right)
$$

for $t \in\left[n^{3 / 4} \mathcal{T}_{k}^{n}, n^{3 / 4} \mathcal{T}_{k+1}^{n}\right)$.

The following theorem is the main result of this section.

Theorem 4.1. Let $\left(\mathbf{X}^{n}(t)\right)$ denote the full trajectory of the particle as defined in (4.1) and let

$$
\iota_{l, u}^{n}:=\inf \left\{t:\left\|\mathbf{X}^{n}(t)\right\| \notin[l, u]\right\} .
$$

For any fixed $l, u \in \mathcal{D}^{\circ}$ with $l<u$, as $n \rightarrow \infty$ we have the following convergence in distribution on $D\left(\mathbb{R}_{+}, \mathbb{R}^{3}\right)$ :

$$
\left(\mathbf{X}^{n}\left(\left(n^{3 / 4} t\right) \wedge \iota_{l, u}^{n}\right)\right)_{t \geq 0} \rightarrow\left(\mathcal{X}\left(\Omega(t) \wedge \tau_{l, u}\right)\right)_{t \geq 0},
$$

where $\mathcal{X}$ is as in Theorem 3.1 and $\Omega$ is the time change given by

$$
\Omega(t):=\mathcal{I}\left(\int_{0}^{\cdot} \frac{d s}{\lambda\left(\mathcal{X}\left(s \wedge \tau_{l, u}\right)\right)}\right)(t)
$$

and $\mathcal{I}$ is the inverse operator defined by $\mathcal{I}(f)(t)=\inf \{s: f(s)>t\}$.

To prove a limit theorem for the full path, we must invert the time process $\left(\mathcal{T}_{k}^{n}\right)_{k \geq 0}$. This is easiest to do if we extend this process to $[0, \infty)$ by linear interpolation:

$$
\mathcal{T}^{n}(s):=\mathcal{T}_{\lfloor s\rfloor}^{n}+(s-\lfloor s\rfloor)\left(\mathcal{T}_{\lfloor s\rfloor+1}^{n}-\mathcal{T}_{\lfloor s\rfloor}^{n}\right), \forall s \in[0, \infty)
$$

Defining $\mathcal{I}(f)(t)=\inf \{s: f(s)>t\}$ we have

$$
\mathbf{X}^{n}\left(n^{3 / 4} \mathcal{T}_{k}^{n}\right)=\mathbf{X}_{\left\lfloor\mathcal{I}\left(\mathcal{T}^{n}\right)\left(\mathcal{T}_{k}^{n}\right)\right\rfloor}^{n}=\mathbf{X}_{k}^{n}
$$

Consequently, we start by examining the convergence of $\left(\mathbf{X}_{\left\lfloor\mathcal{I}\left(\mathcal{T}^{n}\right)(t)\right\rfloor}^{n}\right)_{t \geq 0}$, and then prove that filling in the true values of the path on the intervals $\left[n^{-1} \mathcal{T}_{k}^{n}, n^{-1} \mathcal{T}_{k+1}^{n}\right)$ does not affect the limit. The cutoffs do affect the time change in a technical way. In particular, the stopped process $\left(\mathcal{T}^{n}\left(t \wedge \tau_{l, u}^{n}\right)\right)_{t \geq 0}$ does not have invertible paths. To accommodate this, we use the time change $\Omega_{n}: \mathbb{R}_{+} \rightarrow \mathbb{R}_{+}$defined by

$$
\Omega_{n}(t)=\mathcal{I}\left(\mathcal{T}_{(n \cdot) \wedge \tau_{l, u}^{n}}^{n}+\left(\cdot-\frac{1}{n} \tau_{l, u}^{n}\right)^{+} \frac{1}{\lambda\left(\mathbf{X}^{n}\left(\tau_{l, u}^{n}\right)\right)}\right)(t),
$$


where $\lambda(\mathbf{x})=g(\mathbf{x}) v(\mathbf{x})$. The choice of the particular linear drift is because Theorem 3.1. together with the result in the proof about convergence of hitting times, implies that as $n \rightarrow \infty$

$$
\begin{gathered}
\left(\mathbf{X}_{\lfloor n t\rfloor \wedge \tau_{l, u}^{n}, \mathcal{T}_{(n t) \wedge \tau_{l, u}^{n}}^{n}+\left(t-n^{-1} \tau_{l, u}^{n}\right)^{+}} \frac{1}{\lambda\left(\mathbf{X}^{n}\left(\tau_{l, u}^{n}\right)\right)}\right)_{t \geq 0} \\
\Rightarrow\left(\mathcal{X}_{t \wedge \tau_{l, u}}, \int_{0}^{t} \frac{d s}{\lambda\left(\mathcal{X}_{\left.s \wedge \tau_{l, u}\right)}\right)}\right)_{t \geq 0}
\end{gathered}
$$

The next result allows us to invert the time process.

Lemma 4.2. If $f \in D\left(\mathbb{R}_{+}, \mathbb{R}_{+}\right)$is continuous and strictly increasing with $\lim _{t \rightarrow \infty} f(t)=\infty$, then $\mathcal{I}(f) \in D\left(\mathbb{R}_{+}, \mathbb{R}_{+}\right)$and $\mathcal{I}$ is continuous at $f$.

Proof of Theorem 4.1. By (4.5) and Lemma 4.2 we have

$$
\left(\mathbf{X}_{\lfloor n t\rfloor \wedge \tau_{l, u}^{n}}^{n}, \Omega_{n}(t)\right)_{t \geq 0} \Rightarrow\left(\mathcal{X}_{t \wedge \tau_{l, u}}, \Omega(t)\right)_{t \geq 0} .
$$

Note that the process $(\Omega(t))_{t \geq 0}$ is supported on $C\left(\mathbb{R}_{+}, \mathbb{R}_{+}\right)$and, consequently, the composition map $(f, g) \mapsto f \circ g$ from $D\left(\mathbb{R}_{+}, \mathbb{R}^{3}\right) \times C\left(\mathbb{R}_{+}, \mathbb{R}_{+}\right) \rightarrow D\left(\mathbb{R}_{+}, \mathbb{R}^{3}\right)$ is continuous; see e.g. Bil99, p. 151]. This combined with (4.6) implies that

$$
\left(\mathbf{X}_{\left\lfloor\mathcal{I}\left(\mathcal{T}^{n}\right)(t)\right\rfloor \wedge \tau_{l, u}^{n}}^{n}\right)_{t \geq 0}=\left(\mathbf{X}_{\left\lfloor n \Omega_{n}(t)\right\rfloor \wedge \tau_{l, u}^{n}}^{n}\right)_{t \geq 0} \Rightarrow\left(\mathcal{X}_{\Omega(t) \wedge \tau_{l, u}}\right)_{t \geq 0}
$$

as $n \rightarrow \infty$. For $T>0$ fixed, by (4.3), we have that

$$
\begin{aligned}
& \sup _{0 \leq t \leq T}\left\|\mathbf{X}^{n}\left(n^{3 / 4}\left(t \wedge \mathcal{T}_{\tau_{l, u}^{n}}^{n}\right)\right)-\mathbf{X}_{\left\lfloor\mathcal{I}\left(\mathcal{T}^{n}\right)(t)\right\rfloor \wedge \tau_{l, u}^{n}}^{n}\right\| \\
& \leq \sup _{k \leq\left\lceil n \Omega_{n}\left(T \wedge \mathcal{T}_{\tau_{l, u}^{n} n}^{n}\right)\right\rceil \mathcal{T}_{k-1}^{n} \leq n^{-3 / 4} t \leq \mathcal{T}_{k}^{n}}\left\|\mathbf{y}_{n}\left(\mathbf{X}_{k-1}^{n}, \mathbf{U}_{k-1}, t-n^{3 / 4} \mathcal{T}_{k-1}^{n}\right)-\mathbf{X}_{k-1}^{n}\right\| .
\end{aligned}
$$

Note that

$$
n \Omega_{n}\left(T \wedge \mathcal{T}_{\tau_{l, u}^{n}}^{n}\right) \leq n \Omega_{n}\left(\mathcal{T}_{\tau_{l, u}^{n}}^{n}\right)=\tau_{l, u}^{n}=O(n)
$$

in probability since $n^{-1} \tau_{l, u}^{n} \rightarrow \tau_{l, u}<\infty$ a.s. Thus if we fix $\epsilon>0$, for $n$ sufficiently large we have

$$
\begin{aligned}
& \mathbb{P}\left(\sup _{0 \leq t \leq T}\left\|\mathbf{X}^{n}\left(n^{3 / 4}\left(t \wedge \mathcal{T}_{\tau_{l, u}^{n}}^{n}\right)\right)-\mathbf{X}_{\left\lfloor\mathcal{I}\left(\mathcal{T}^{n}\right)(t)\right\rfloor \wedge \tau_{l, u}^{n}}^{n}\right\|>\epsilon\right) \\
& \quad \leq \epsilon+\mathbb{P}\left(\sup _{k \leq n^{2}} \sup _{\mathcal{T}_{k-1}^{n} \leq n^{-3 / 4} t \leq \mathcal{T}_{k}^{n}}\left\|\mathbf{y}_{n}\left(\mathbf{X}_{k-1}^{n}, \mathbf{U}_{k-1}, t-n^{3 / 4} \mathcal{T}_{k-1}^{n}\right)-\mathbf{X}_{k-1}^{n}\right\|\right. \\
& \left.\times \mathbb{1}_{\left\{k \leq \tau_{l, u}^{n}+1\right\}}>\epsilon\right) .
\end{aligned}
$$

The probability on the right-hand side can be made arbitrarily small by observing that, by Corollary 2.5. for $C>0$,

$$
\begin{aligned}
& \mathbb{P}\left(n^{3 / 4}\left(\mathcal{T}_{k}^{n}-\mathcal{T}_{k-1}^{n}\right)>C \mid\left\|\mathbf{X}_{k-1}^{n}\right\| \in[l, u]\right) \\
& \quad \leq \sup _{(\mathbf{x}, \mathbf{u}):\|\mathbf{x}\| \in[l, u], \mathbf{u} \in \mathbf{S}^{2}} \mathbb{P}\left(N^{(n)}(\mathbf{x}, \mathbf{u})>C\right)=o\left(n^{k}\right)
\end{aligned}
$$


in probability for every $k \geq 1$ and by Lemma 2.3. if $n$ is large enough, then

$$
\sup _{(\mathbf{x}, \mathbf{u}, t) \in\{\mathbf{x}:\|\mathbf{x}\| \in[l, u]\} \times \mathbf{S}^{2} \times[0, C]}\left\|\mathbf{y}_{n}(\mathbf{x}, \mathbf{u}, t)-\mathbf{x}\right\|<\epsilon .
$$

Therefore

$$
\left(\mathbf{X}^{n}\left(n^{3 / 4}\left(t \wedge \mathcal{T}_{\tau_{l, u}^{n}}^{n}\right)\right)\right)_{t \geq 0} \Rightarrow\left(\mathcal{X}_{\Omega(t) \wedge \tau_{l, u}}\right)_{t \geq 0}
$$

as $n \rightarrow \infty$ and the fact that

$$
\left(\mathbf{X}^{n}\left(\left(n^{3 / 4} t\right) \wedge \iota_{l, u}^{n}\right)\right)_{t \geq 0} \Rightarrow\left(\mathcal{X}_{\Omega(t) \wedge \tau_{l, u}}\right)_{t \geq 0}
$$

as $n \rightarrow \infty$ follow from path continuity and observing that $\iota_{l, u}^{n} \leq n^{3 / 4} \mathcal{T}_{\tau_{l, u}^{n}}^{n}$.

\section{Classifying the Boundaries of $\mathcal{D}$}

In this section we give conditions under which the boundary points of $\mathcal{D}$ are inaccessible, meaning the boundary points cannot be reached in finite time. These results can then be used to remove the stopping at $u$ and/or $l$ in Theorem 1.4.

Suppose we have a regular diffusion $X$ with state space the interval $(\ell, r)$. Every diffusion has two basic characteristics: the speed measure $m(d x)$ and the scale function $s(x)$.

We assume the infinitesimal generator $G: \mathcal{D}(G) \mapsto \mathcal{C}_{b}(I)$ of $X$ is a second order differential operator

$$
G f(x)=\frac{1}{2} \sigma^{2}(x) \partial_{x x} f(x)+\mu(x) \partial_{x} f(x),
$$

where $\sigma, \mu \in C(I)$ and $\sigma^{2}(x)>0$ for all $x \in I$. Let $B(x):=\int_{a}^{x} 2 \sigma^{-2}(y) \mu(y) d y$ for some arbitrary (fixed) $a \in I$. Then it is well known that:

- The speed measure is absolutely continuous with respect to Lebesgue measure and has density

$$
m^{\prime}(x)=2 \sigma^{-2}(x) e^{B(x)} .
$$

- The scale function has density

$$
s^{\prime}(x)=e^{-B(x)} .
$$

The domain $\mathcal{D}(G)$ consists of all functions in $\mathcal{C}_{b}(I)$ such that $G f \in \mathcal{C}_{b}(I)$ together with the appropriate boundary conditions.

The boundary point $\ell$ is called accessible when

$$
\int_{\ell}^{x}\left(\int_{y}^{x} m^{\prime}(\eta) d \eta\right) s^{\prime}(y) d y<\infty
$$

and inaccessible when

$$
\int_{\ell}^{x}\left(\int_{y}^{x} m^{\prime}(\eta) d \eta\right) s^{\prime}(y) d y=\infty .
$$

Similarly, one can classify the boundary $r$.

As we have shown above in Corollary 3.2 the one-dimensional diffusion defining the limiting radial process has generator $\mathcal{G}_{r}$ that acts on compactly supported 
functions in $C^{2}\left(\mathcal{D}^{\circ}\right)$ as

$$
\begin{aligned}
\mathcal{G}_{r} f(\rho) & :=\mu_{r}(\rho) f^{\prime}(\rho)+\frac{\sigma_{r}^{2}(\rho)}{2} f^{\prime \prime}(\rho) \\
& =\frac{1}{3 g^{2}(\rho)}\left(-\frac{g^{\prime}(\rho)}{g(\rho)}+\frac{1}{\rho}-\frac{\partial_{r} \mathcal{U}(\rho)}{(E-\mathcal{U}(\rho))}\right) f^{\prime}(\rho)+\frac{1}{3 g^{2}(\rho)} f^{\prime \prime}(\rho) .
\end{aligned}
$$

If $a \in \mathcal{D}^{\circ}$, then the density of the scale function will be

$$
\begin{aligned}
s^{\prime}(y) & =\exp \left(-\int_{a}^{y} \frac{2 \mu_{r}(\rho)}{\sigma_{r}^{2}(\rho)} d \rho\right) \\
& =\exp \left(\int_{a}^{y}\left(\frac{g^{\prime}(\rho)}{g(\rho)}-\frac{1}{\rho}+\frac{\partial_{r} \mathcal{U}(\rho)}{(E-\mathcal{U}(\rho))}\right) d \rho\right) \\
& =\exp (\ln (g(y))-\ln (y)-\ln (E-\mathcal{U}(y))-C) \\
& =\exp \left(\ln \left(\frac{g(y)}{y(E-\mathcal{U}(y))}\right)-C\right) \\
& =\frac{a(E-\mathcal{U}(a))}{g(a)}\left(\frac{g(y)}{y(E-\mathcal{U}(y))}\right),
\end{aligned}
$$

where $C:=\ln \left(\frac{g(a)}{a(E-\mathcal{U}(a))}\right)$. As a result, if we fix an arbitrary $c \in \mathcal{D}^{\circ}$ the scale function will be given by

$$
s(x)=\int_{c}^{x} s^{\prime}(y) d y=\frac{a(E-\mathcal{U}(a))}{g(a)} \int_{c}^{x}\left(\frac{g(y)}{y(E-\mathcal{U}(y))}\right) d y .
$$

The speed measure density for the radial diffusion will be

$$
m^{\prime}(x)=\frac{2}{\sigma_{r}^{2}(x) s^{\prime}(x)}=\frac{2 g(a)}{a(E-\mathcal{U}(a))} g(x) x(E-\mathcal{U}(x)) .
$$

One can then find the speed measure by setting

$$
m(J):=\int_{J} m^{\prime}(x) d x=\frac{2 g(a)}{a(E-\mathcal{U}(a))} \int_{J} g(x) x(E-\mathcal{U}(x)) d x
$$

for any Lebesgue measurable $J \subseteq D^{\circ}$.

Remark 5.1. If $s(\ell)=-\infty$, then $\ell$ cannot be reached in finite time and is therefore inaccessible. Similarly, if $s(r)=\infty$, then $r$ is inaccessible.

Proposition 5.2. Let $D^{\circ}=\left(h_{-}, h_{+}\right)$.

(i) Suppose that there exists $\alpha \geq 1$ such that when $y \downarrow h_{-}$

$$
\frac{1}{y(E-\mathcal{U}(y))}=\mathcal{O}\left(\left(y-h_{-}\right)^{-\alpha}\right) \text {. }
$$

Then $h_{-}$is inaccessible.

(ii) Suppose that there exists $\alpha<1$ such that when $y \downarrow h_{-}$

$$
\frac{1}{y(E-\mathcal{U}(y))}=\mathcal{O}\left(\left(y-h_{-}\right)^{-\alpha}\right) \text {. }
$$

Then $h_{-}$is accessible.

In particular, if $h_{-}=0$ and $|\mathcal{U}(0)|<\infty$, then 0 is inaccessible. Analogous results hold for $h_{+}$. 
Proof. The main parts of the proposition follow easily from (5.2) and Remark 5.1] If $h_{-}=0$ and $|\mathcal{U}(0)|<\infty$, then, since by assumption $E>\mathcal{U}(0)$, we have

$$
\frac{1}{y(E-\mathcal{U}(y))}=\mathcal{O}\left(y^{-1}\right)
$$

5.1. Constant force. Suppose we have a constant force field

$$
\mathcal{U}(x)=C x
$$

and $\mathcal{D}=[0, h]$ for some $h>0$. We know that the speed has to be zero at $h$ so

$$
E-\mathcal{U}(h)=E-C h=0 .
$$

This forces $E=C h$ and thus

$$
\frac{1}{y(E-\mathcal{U}(y))}=\frac{1}{y C(h-y)} .
$$

By Proposition 5.2 both 0 and $h$ are inaccessible boundary points.

5.2. Newtonian gravity. We consider the case when the potential is that of a gravitational force directed towards the origin. That is, the potential function is given by

$$
\mathcal{U}(\rho)=-\frac{k}{\rho}
$$

for some constant $k>0$. Suppose the total energy of the particle is positive, $E>0$. We have

$$
s(x)=\frac{(E a+k) a}{g(a)} \int_{c}^{x}\left(\frac{g(y)}{(E y+k) y}\right) d y .
$$

Since $g$ is bounded above and bounded away from 0 on $\mathbb{R}_{+}$, for $y \downarrow 0$,

$$
\frac{g(y)}{\sqrt{(E y+k) y}}=\mathcal{O}\left(\frac{1}{y}\right)
$$

which implies $\lim _{x \downarrow 0} s(x)=-\infty$. For $y \uparrow \infty$ one has

$$
\frac{g(y)}{(E y+k) y}=\mathcal{O}\left(\frac{1}{y^{2}}\right)
$$

so that $\lim _{x \uparrow \infty} s(x)=\infty$. By Proposition 5.2 the boundary points 0 and $\infty$ are inaccessible.

\section{Higher Dimensions AND GENERAL POTENTIALS}

Although our model was motivated by the three-dimensional Lorentz gas, the process with uniform reflection direction makes sense in any dimension and our methods apply essentially without change. In this section we explain how our results could be generalized to higher dimensions and to nonspherically symmetric potentials. Consider a particle in $\mathbb{R}^{d}$ for $d \in \mathbb{N}$ under the influence of a force field with potential energy $\mathcal{U}(\boldsymbol{x})$. Let $S^{d-1}$ be the unit sphere in $\mathbb{R}^{d}$. In this model upon reflection at $\boldsymbol{x}=\left(x_{1}, \ldots, x_{d}\right)$, the particle starts its path in the uniform direction $\boldsymbol{u}=\left(u_{1}, \ldots, u_{d}\right) \in S^{d-1}$ and then travels under the influence of $\mathcal{U}(\boldsymbol{x})$. The only difference is that some of the constants change because we are integrating $\boldsymbol{u}$ over $\mathbf{S}^{d-1}$ instead of $\mathbf{S}^{2}$. Using the fact that if $\boldsymbol{u}=\left(u_{1}, \ldots, u_{d}\right)$ is uniformly distributed on $\mathbf{S}^{d-1}$, then $\mathbb{E} u_{i}^{2}=\frac{1}{d}$ for all $i \in\{1, \ldots, d\}$ yields the following results. 
Let $\left(\left(\mathbf{X}_{k}^{n}, \mathcal{T}_{k}^{n}\right)\right)_{k \geq 0}$ be the free path Markov chain, where $\mathbf{X}_{k}^{n}$ is the location of the particle at the time of the $k$ th reflection and $n^{3 / 4} \mathcal{T}_{k}^{n}$ is the time of the $k$ th collision.

Theorem 6.1. Let

$$
\mu_{n}(\mathbf{x}, t):=n \mathbb{E}\left[\left(\mathbf{X}_{1}^{n}, \mathcal{T}_{1}^{n}\right)-(\mathbf{x}, t) \mid\left(\mathbf{X}_{0}^{n}, \mathcal{T}_{0}^{n}\right)=(\mathbf{x}, t)\right]
$$

be the scaled drift of $\left(\mathbf{X}_{k}^{n}, \mathcal{T}_{k}^{n}\right)_{k \geq 0}$, and let $S$ be a compact subset of $\mathcal{D}^{\circ}$. Suppose the equivalents of Assumptions (A1)-(A5) are satisfied. Then

$\lim _{n \rightarrow \infty} \sup _{(\mathbf{x}, t):\|\mathbf{x}\| \in S, t \geq 0}\left|\mu_{n}(\mathbf{x}, t)-\left(\frac{-1}{d g(\mathbf{x})^{2}}\left[\frac{(d-1) \nabla \mathcal{U}(\mathbf{x})}{m v(\mathbf{x})^{2}}+\frac{\nabla g(\mathbf{x})}{g(\mathbf{x})}\right], \frac{1}{g(\mathbf{x}) v(\mathbf{x})}\right)\right|=0$

uniformly on $\{\mathbf{x}:\|\mathbf{x}\| \in S\} \times[0, \infty)$. Furthermore, let

$$
\begin{aligned}
\sigma_{n, i j}^{2}(\mathbf{x}, t) & :=n \mathbb{E}\left[\left(X_{1, i}^{n}-x_{i}\right)\left(X_{1, j}^{n}-x_{j}\right) \mid\left(\mathbf{X}_{0}^{n}, \mathcal{T}_{0}^{n}\right)=(\mathbf{x}, t)\right], \\
\sigma_{n, i t}^{2}(\mathbf{x}, t) & :=n \mathbb{E}\left[\left(X_{1, i}^{n}-x_{i}\right)\left(\mathcal{T}_{1}^{n}-t\right) \mid\left(\mathbf{X}_{0}^{n}, \mathcal{T}_{0}^{n}\right)=(\mathbf{x}, t)\right] \\
\sigma_{n, t}^{2}(\mathbf{x}, t) & :=n \mathbb{E}\left[\left(\mathcal{T}_{1}^{n}-t\right)^{2} \mid\left(\mathbf{X}_{0}^{n}, \mathcal{T}_{0}^{n}\right)=(\mathbf{x}, t)\right] .
\end{aligned}
$$

Then

(1) $\lim _{n \rightarrow \infty} \sup _{(\mathbf{x}, t):\|\mathbf{x}\| \in S, t \geq 0}\left|\sigma_{n, i j}^{2}(\mathbf{x}, t)-\frac{d-1}{d g(\mathbf{x})^{2}} \delta_{i j}\right|=0$, where $\delta_{i j}=\mathbb{1}\{i=j\}$.

(2) $\lim _{n \rightarrow \infty} \sup _{(\mathbf{x}, t):\|\mathbf{x}\| \in S, t>0} \sigma_{n, i t}^{2}(\mathbf{x}, t)=0$.

(3) $\lim _{n \rightarrow \infty} \sup _{(\mathbf{x}, t):\|\mathbf{x}\| \in S, t \geq 0} \sigma_{n, t}^{2}(\mathbf{x}, t)=0$.

Theorem 6.2. Let $(\mathscr{X}(t), t \geq 0)$ be a diffusion on $\mathcal{D} \subset \mathbb{R}^{d}$ whose generator $\mathscr{G}$ acts on functions $f \in C^{2}(\mathcal{D})$ with compact support in $C^{2}\left(\mathcal{D}^{\circ}\right)$ by

$$
\mathscr{G} f(\boldsymbol{x})=\frac{v(\boldsymbol{x})}{d g(\boldsymbol{x})} \Delta f(\boldsymbol{x})-\frac{v(\boldsymbol{x})}{g(\boldsymbol{x})}\left(\frac{(d-1) \nabla \mathcal{U}(\boldsymbol{x})}{d m v^{2}(\boldsymbol{x})}+\frac{\nabla g(\boldsymbol{x})}{d g(\boldsymbol{x})}\right) \cdot \nabla f(\boldsymbol{x}) .
$$

Let $\left(\mathbf{X}^{n}(t), t \geq 0\right)$ be the trajectory of the particle and assume the equivalents of Assumptions (A1)-(A5) above hold. Fix a set $\mathcal{J} \subset \mathcal{D}^{\circ}$ and define

$$
\iota_{\mathcal{J}}^{n}=\inf \left\{t \geq 0: \mathbf{X}^{n}(t) \notin \mathcal{J}\right\}
$$

and

$$
\tau_{\mathcal{J}}=\inf \{t \geq 0: \mathscr{X}(t) \notin \mathcal{J}\} .
$$

Then as $n \rightarrow \infty$ we have the following convergence in distribution:

$$
\left(\mathbf{X}^{n}\left(n^{3 / 4} t \wedge \iota_{\mathcal{J}}^{n}\right), t \geq 0\right) \rightarrow\left(\mathscr{X}\left(t \wedge \tau_{\mathcal{J}}\right), t \geq 0\right) .
$$

Futhermore, if the boundary of $\mathcal{D}$ is inaccessible, then we can remove the stopping in the above.

\section{ACKNowledgments}

The authors thank Simon Griffiths, Cristina Costantini, and Steve Evans for very helpful discussions. 


\section{REFERENCES}

[ADBLP10] B. Aguer, S. De Bièvre, P. Lafitte, and P. E. Parris, Classical motion in force fields with short range correlations, J. Stat. Phys. 138 (2010), no. 4-5, 780-814, DOI 10.1007/s10955-009-9898-7. MR2594921

[AB99] G. Allaire and G. Bal, Homogenization of the criticality spectral equation in neutron transport (English, with English and French summaries), M2AN Math. Model. Numer. Anal. 33 (1999), no. 4, 721-746, DOI 10.1051/m2an:1999160. MR:1726482

[BR00] G. Bal and L. Ryzhik, Diffusion approximation of radiative transfer problems with interfaces, SIAM J. Appl. Math. 60 (2000), no. 6, 1887-1912, DOI 10.1137/S0036139999352080. MR 1763308

[BFS00] J. Banasiak, G. Frosali, and G. Spiga, Inelastic scattering models in transport theory and their small mean free path analysis, Math. Methods Appl. Sci. 23 (2000), no. 2, 121-145, DOI 10.1002/(SICI)1099-1476(20000125)23:2〈121::AIDMMA107〉3.3.CO;2-X. MR 1738348

[vBM05] H. van Beijeren and O. Mülken, Thermodynamic formalism for the Lorentz gas with open boundaries in d dimensions, Phys. Rev. E 71 (2005), 036213.

[BAD96] N. Ben Abdallah and P. Degond, On a hierarchy of macroscopic models for semiconductors, J. Math. Phys. 37 (1996), no. 7, 3306-3333, DOI 10.1063/1.531567. MR.1401227

[BLP79] A. Bensoussan, J.-L. Lions, and G. C. Papanicolaou, Boundary layers and homogenization of transport processes, Publ. Res. Inst. Math. Sci. 15 (1979), no. 1, 53-157, DOI $10.2977 /$ prims/1195188427. MR533346

[BHHP96] N. Berglund, Alex Hansen, E. H. Hauge, and J. Piasecki, Can a local repulsive potential trap an electron?, Phys. Rev. Lett. 77 (1996), 2149-2153.

[BCDS06] B. Berkowitz, A. Cortis, M. Dentz, and H. Scher, Modeling non-fickian transport in geological formations as a continuous time random walk, Reviews of Geophysics 44 (2006), no. 2.

[Bi199] P. Billingsley, Convergence of probability measures, 2nd ed., Wiley Series in Probability and Statistics: Probability and Statistics, John Wiley \& Sons, Inc., New York, 1999. A Wiley-Interscience Publication. MR:1700749

[BHPH01] A. V. Bobylev, A. Hansen, J. Piasecki, and E. H. Hauge, From the Liouville equation to the generalized Boltzmann equation for magnetotransport in the 2D Lorentz model, J. Statist. Phys. 102 (2001), no. 5-6, 1133-1150, DOI 10.1023/A:1004880010020. MR:1830442

[BMHH97] A. V. Bobylev, F. A. Maaø, A. Hansen, and E. H. Hauge, There is more to be learned from the Lorentz model, J. Statist. Phys. 87 (1997), no. 5-6, 1205-1228, DOI 10.1007/BF02181280. MR.1470650

[BZ07] F. P. Boca and A. Zaharescu, The distribution of the free path lengths in the periodic two-dimensional Lorentz gas in the small-scatterer limit, Comm. Math. Phys. 269 (2007), no. 2, 425-471, DOI 10.1007/s00220-006-0137-7. MR2274553

[BGW98] J. Bourgain, F. Golse, and B. Wennberg, On the distribution of free path lengths for the periodic Lorentz gas, Comm. Math. Phys. 190 (1998), no. 3, 491-508, DOI $10.1007 / \mathrm{s} 002200050249$. MR 1600299

[BCLDM02] C. Buet, S. Cordier, B. Lucquin-Desreux, and S. Mancini, Diffusion limit of the Lorentz model: asymptotic preserving schemes, M2AN Math. Model. Numer. Anal. 36 (2002), no. 4, 631-655, DOI 10.1051/m2an:2002028. MR.1932307

[BR14] K. Burdzy and D. Rizzolo, A Lorentz model with variable density in a gravitational field: limit theorems and recurrence, ArXiv e-prints (2014), Available at http:// arxiv.org/abs/1310.7312.

[CG03] E. Caglioti and F. Golse, On the distribution of free path lengths for the periodic Lorentz gas. III, Comm. Math. Phys. 236 (2003), no. 2, 199-221, DOI 10.1007/s00220-003-0825-5. MR 1981109

[CD09] N. Chernov and D. Dolgopyat, The Galton board: limit theorems and recurrence, J. Amer. Math. Soc. 22 (2009), no. 3, 821-858, DOI 10.1090/S0894-0347-08-00626-7. MR2505302 
[Cos91] C. Costantini, Diffusion approximation for a class of transport processes with physical reflection boundary conditions, Ann. Probab. 19 (1991), no. 3, 1071-1101. MR.112407

[Deg98] P. Degond, A model of near-wall conductivity and its application to plasma thrusters, SIAM J. Appl. Math. 58 (1998), no. 4, 1138-1162 (electronic), DOI 10.1137/S0036139996300897. MR 1620406

[DR04] L. Desvillettes and V. Ricci, Non-Markovianity of the Boltzmann-Grad limit of a system of random obstacles in a given force field, Bull. Sci. Math. 128 (2004), no. 1, 39-46, DOI 10.1016/j.bulsci.2003.09.003. MR2033100

[Det14] C. P. Dettmann, Diffusion in the Lorentz gas, Commun. Theor. Phys. (Beijing) 62 (2014), no. 4, 521-540, DOI 10.1088/0253-6102/62/4/10. MR3363162

[DLLS13] M. Dobson, F. Legoll, T. Lelièvre, and G. Stoltz, Derivation of Langevin dynamics in a nonzero background flow field, ESAIM Math. Model. Numer. Anal. 47 (2013), no. 6, 1583-1626, DOI 10.1051/m2an/2013077. MR.3110489

[DK09] D. Dolgopyat and L. Koralov, Motion in a random force field, Nonlinearity 22 (2009), no. 1, 187-211, DOI 10.1088/0951-7715/22/1/010. MR2470270

[DGL81] D. Dürr, S. Goldstein, and J. L. Lebowitz, A mechanical model of Brownian motion, Comm. Math. Phys. 78 (1980/81), no. 4, 507-530. MR606461

[EK86] S. N. Ethier and T. G. Kurtz, Markov processes: Characterization and convergence, Wiley Series in Probability and Mathematical Statistics: Probability and Mathematical Statistics, John Wiley \& Sons, Inc., New York, 1986. MR838085

[Gal78] G. Gallavotti, Rigorous theorey of the Boltzmann equation in the Lorentz gas, updated reprint of Nota Interna n.358, Instituta di Fisica, University of Rome, 1972.

[GW00] F. Golse and B. Wennberg, On the distribution of free path lengths for the periodic Lorentz gas. II (English, with English and French summaries), M2AN Math. Model. Numer. Anal. 34 (2000), no. 6, 1151-1163, DOI 10.1051/m2an:2000121. MR 1812731

$\left[\mathrm{HLB}^{+} 09\right]$ L. Han, B. H. Lui, S. Blumberg, J. F. Beausang, P. C. Nelson, and R. Phillips, Calibration of tethered particle motion experiments, Mathematics of DNA structure, function and interactions, IMA Vol. Math. Appl., vol. 150, Springer, New York, 2009, pp. 123-138, DOI 10.1007/978-1-4419-0670-0_6. MR2762649

[HW13] K.-C. Huang and R. J. White, Random walk on a leash: A simple single-molecule diffusion model for surface-tethered redox molecules with flexible linkers, J. Am. Chem. Soc. 135 (2013), no. 34, 12808-12817.

[JS03] J. Jacod and A. N. Shiryaev, Limit theorems for stochastic processes, 2nd ed., Grundlehren der Mathematischen Wissenschaften [Fundamental Principles of Mathematical Sciences], vol. 288, Springer-Verlag, Berlin, 2003. MR 1943877

[Kal02] O. Kallenberg, Foundations of modern probability, 2nd ed., Probability and its Applications (New York), Springer-Verlag, New York, 2002. MR 1876169

[KR06] T. Komorowski and L. Ryzhik, Diffusion in a weakly random Hamiltonian flow, Comm. Math. Phys. 263 (2006), no. 2, 277-323, DOI 10.1007/s00220-005-1500-9. MR2207646

[Kur73] T. G. Kurtz, A limit theorem for perturbed operator semigroups with applications to random evolutions, J. Functional Analysis 12 (1973), 55-67. MR0365224

[KS98] A. Kuzmany and H. Spohn, Magnetotransport in the two-dimensional Lorentz gas, Phys. Rev. E 57 (1998), 5544-5553.

[Lor05] H. A. Lorentz, The motion of electrons in metallic bodies $i$, KNAW, Proceedings 7 (1905), 438-453.

[MS10] J. Marklof and A. Strömbergsson, The distribution of free path lengths in the periodic Lorentz gas and related lattice point problems, Ann. of Math. (2) 172 (2010), no. 3, 1949-2033, DOI 10.4007/annals.2010.172.1949. MR.2726104

[MS11a] J. Marklof and A. Strömbergsson, The Boltzmann-Grad limit of the periodic Lorentz gas, Ann. of Math. (2) 174 (2011), no. 1, 225-298, DOI 10.4007/annals.2011.174.1.7. MR2811599

[MS11b] J. Marklof and A. Strömbergsson, The periodic Lorentz gas in the Boltzmann-Grad limit: asymptotic estimates, Geom. Funct. Anal. 21 (2011), no. 3, 560-647, DOI 10.1007/s00039-011-0116-9. MR2810859

[MS14a] J. Marklof and A. Strömbergsson, Free path lengths in quasicrystals, Comm. Math. Phys. 330 (2014), no. 2, 723-755, DOI 10.1007/s00220-014-2011-3. MR3223485 
[MS14b] J. Marklof and A. Strömbergsson, Power-law distributions for the free path length in Lorentz gases, J. Stat. Phys. 155 (2014), no. 6, 1072-1086, DOI 10.1007/s10955014-0935-9. MR3207729

[MS04] M. M. Meerschaert and H.-P. Scheffler, Limit theorems for continuous-time random walks with infinite mean waiting times, J. Appl. Probab. 41 (2004), no. 3, 623-638. MR2074812

[MS14c] M. M. Meerschaert and P. Straka, Semi-Markov approach to continuous time random walk limit processes, Ann. Probab. 42 (2014), no. 4, 1699-1723, DOI 10.1214/13AOP905. MR 3262490

[MK00] R. Metzler and J. Klafter, The random walk's guide to anomalous diffusion: a fractional dynamics approach, Phys. Rep. 339 (2000), no. 1, 77, DOI 10.1016/S03701573(00)00070-3. MR:1809268

[MvB04] O. Mülken and H. van Beijeren, Thermodynamic formalism for field-driven Lorentz gases, Phys. Rev. E 69 (2004), 046203.

[Pap75] G. C. Papanicolaou, Asymptotic analysis of transport processes, Bull. Amer. Math. Soc. 81 (1975), 330-392. MR0362523

[PW79] J. Piasecki and E. Wajnryb, Long-time behavior of the Lorentz electron gas in a constant, uniform electric field, Journal of Statistical Physics 21 (1979), no. 5, 549559 (English).

[RT99] K. Ravishankar and L. Triolo, Diffusive limit of the Lorentz model with a uniform field starting from the Markov approximation, Markov Process. Related Fields 5 (1999), no. 4, 385-421. MR 1734241

[SBMB03] R. Schumer, D. A. Benson, M. M. Meerschaert, and B. Baeumer, Fractal mobile/immobile solute transport, Water Resources Research 39 (2003), no. 10.

[Spo78] H. Spohn, The Lorentz process converges to a random flight process, Comm. Math. Phys. 60 (1978), no. 3, 277-290. MR0496299

[Spo88] H. Spohn, Kinetic equations from Hamiltonian dynamics: the Markovian approximations, Kinetic theory and gas dynamics, CISM Courses and Lectures, vol. 293, Springer, Vienna, 1988, pp. 183-211.

[Vys06] V. Vysotsky, A functional limit theorem for the position of a particle in a Lorentz type model, Markov Process. Related Fields 12 (2006), no. 4, 767-790. MR2284402

$\left[\mathrm{WZL}^{+}{ }_{14}\right]$ J. Wang, J. Zhou, L.-J. Lv, W.-Y. Qiu, and F.-Y. Ren, Heterogeneous memorized continuous time random walks in an external force fields, J. Stat. Phys. 156 (2014), no. 6, 1111-1124, DOI 10.1007/s10955-014-1046-3. MR.3240872

[Wen12] B. Wennberg, Free path lengths in quasi crystals, J. Stat. Phys. 147 (2012), no. 5, 981-990, DOI 10.1007/s10955-012-0500-3. MR2946632

[WW05] R. J. White and H. S. White, A random walk through election-transfer kinetics, Anal. Chem. 77 (2005), no. 11, 214A-220A.

[dWB04] A. S. de Wijn and H. van Beijeren, Lyapunov spectrum of the many-dimensional dilute random Lorentz gas, Phys. Rev. E 70 (2004), 036209.

[WE82] D. R. Wilkinson and S. F. Edwards, Spontaneous interparticle percolation, Proc. Roy. Soc. London Ser. A 381 (1982), no. 1780, 33-51, DOI 10.1098/rspa.1982.0057. MR 661715

Department of Statistics, University of Oxford, 24-29 St Giles, Oxford OX1 3LB, UNiTED Kingdom

E-mail address: hening@stats.ox.ac.uk

Department of Mathematical Sciences, University of Delaware, 15 Orchard Road, NeWARK, DelaWare 19716

E-mail address: drizzolo@udel.edu

Department of Mathematics, University of California, 970 Evans Hall \#3840, BerkeLEY, CALIFORNiA 94720-3840

E-mail address: ewayman@gmail.com 